\title{
Large-Signal Modeling of GaN Devices for Designing High Power Amplifiers of Next Generation Wireless Communication Systems
}

\author{
Anwar Jarndal \\ Hodeidah University \\ Yemen
}

\section{Introduction}

An excellent candidate for fabrication of high-power amplifiers (HPAs) for next-generation wireless communication systems is a GaN HEMT. It has high sheet carrier density and high saturation electron velocity, which produce high output power. It also has high electron mobility, which is largely responsible for low on-resistance value which enhances highpower-added efficiency. As a result of $\mathrm{GaN}$ as a wideband material, the GaN HEMTs can achieve very high breakdown voltage and very high current density, and they can sustain very high channel-operating temperature. Furthermore, a possible epitaxial growth on silicon carbide substrate, which has excellent thermal properties, makes this device optimal for high-power RF applications. The past decade saw rapid progress in the development of GaN HEMTs with a focus on its power performance (Eastman et al., 2001). However, despite the high output power of this device, current dispersion is the biggest obstacle in obtaining reproducible power performance (Vetury et al., 2001); (Meneghesso et al., 2004). Designing an HPA based on the GaN HEMTs requires an accurate large-signal model for this device. This model should account for the current dispersion and temperaturedependent performance in addition to other high-power-stimulated effects like gate forward and breakdown phenomenon. In particular, the model should be able to predict intermodulation distortion (IMD), which is very important for the analysis of the HPA nonlinearity. In the last decade different models have been developed for GaN HEMTs. The analytical models reported in (Green et al., 2000) and (Lee \& Webb, 2004) can simulate the fundamental output power including the current dispersion and thermal characteristics of the GaN HEMTs. However, these models have poor IMD-prediction capabilities. In another reported model (Raay et al., 2003), no IMD simulation has been presented. The model published in (Cabral et al., 2004) has been optimized for IMD simulation, but it does not account for the current dispersion or the temperature-dependent characteristics. This chapter addresses the development of a large-signal model for GaN HEMTs, which can simulate all of the mentioned effects in an efficient manner. First, a small-signal model that will be used as a basis for constructing the large-signal model will be described. Detailed steps for extraction of the small-signal model parameters will be presented. Large-signal 
modeling and extraction procedures will also be explained. Finally, the developed largesignal model will be validated by comparing its simulations with measurements.

\section{GaN HEMT}

The general structure of the investigated devices is shown in Figure 1. The GaN HEMT structure was grown on $\mathrm{SiC}$ 2-inch wafers using Metal-Organic-Chemical-VapourDeposition (MOCVD) technology (Lossya et al., 2002). This substrate provides an excellent thermal conductivity of $3.5 \mathrm{~W} / \mathrm{cm}$, which is an order of magnitude higher than that of sapphire. The epitaxial growth structure starts with the deposition of a $500 \mathrm{~nm}$ thick graded AlGaN layer on the substrate to reduce the number of threading dislocations in the GaN buffer layer due to the lattice mismatch between $\mathrm{GaN}$ and $\mathrm{SiC}$ layers. These threading dislocations enhance buffer traps and hence the associated drain-current dispersion (Hansen et al., 1998). A $2.7 \mu \mathrm{m}$ thick highly insulating GaN buffer layer is then deposited to get lower background carrier concentration, which accordingly results in increased electron mobility in the above unintentionally doped layers. The buffer layer is followed by a $3 \mathrm{~nm}$ $\mathrm{Al}_{0.25} \mathrm{Ga}_{0.75} \mathrm{~N}$ spacer, $12 \mathrm{~nm}$ Si-doped $\mathrm{Al}_{0.25} \mathrm{Ga}_{0.75} \mathrm{~N}$ supply layer $\left(5 \times 1018 \mathrm{~cm}^{-3}\right)$, and $10 \mathrm{~nm}$ $\mathrm{Al}_{0.25} \mathrm{Ga}_{0.75} \mathrm{~N}$ barrier layer. The spontaneous and piezoelectric polarizations of the $\mathrm{Al}_{0.25} \mathrm{Ga}_{0.75} \mathrm{~N}$ layers form a two-dimensional electron gas (2DEG) at the AlGaN/GaN interface (Ambacher et al., 1999). The spacer layer is included to reduce the ionized-impurity scattering that deteriorates electron mobility in the 2DEG. The whole structure is capped with a $5 \mathrm{~nm}$ thick GaN layer to increase the effective Schottky barrier, which improves the breakdown characteristics and decreases the gate leakage. The measured 2DEG electron density and mobility, at room temperature, are $7.8 \times 10^{12} \mathrm{~cm}^{-2}$ and $1400 \mathrm{~cm}^{2} / \mathrm{Vs}$ (Lossya et al., 2002). Device fabrication is accomplished using $0.5-\mu \mathrm{m}$ stepper lithography, which results in an excellent homogeneity of the electrical properties over the wafer (Lossy et al., 2001).

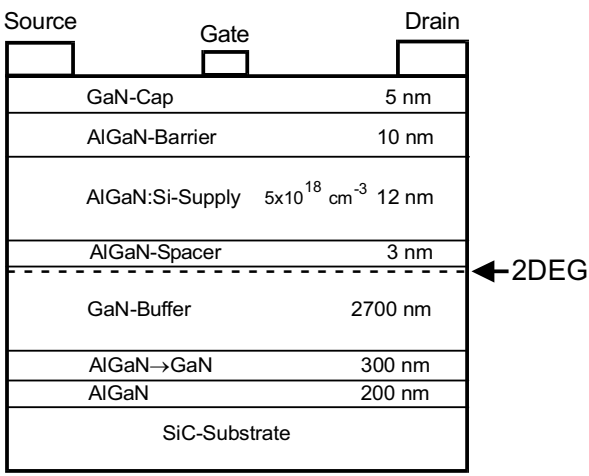

Fig. 1. Epitaxial layer structure of GaN HEMT.

Source and drain ohmic contacts have a metallization consisting of $\mathrm{Ti} / \mathrm{Al} / \mathrm{Ti} / \mathrm{Au} / \mathrm{WSiN}$ $(10 / 50 / 25 / 30 / 120 \mathrm{~nm})$ with improved edge and surface morphology. Due to the properties of the WSiN sputter deposition process, the Ti/Al/Ti/Au layers, which are deposited by ebeam evaporation, are totally embedded. The source and drain contacts are then rapidly thermal-annealed at $850{ }^{\circ} \mathrm{C}$. The contact resistance is analyzed by Thermal Lens Microscope 
(TLM) measurements with respect to thickness and composition of the different metallization layers at different temperatures. The contact resistance is determined to be 0.25-0.5 $\Omega \mathrm{mm}$ under these conditions (Lossya et al., 2002). Gate contacts are made from a $\mathrm{Pt} / \mathrm{Au}$ metallization, and a gate length of $0.5 \mu \mathrm{m}$ is obtained using stepper lithography. Additionally, devices with gate length less than $0.3 \mu \mathrm{m}$ are written using a shaped electron beam tool (ZBA23-40kV) (Lossyb et al., 2002). SiN passivation layer is then deposited to reduce the surface trapping induced drain-current dispersion. Field plate connected to the gate, at the gate pad, and deposited over the passivation layer was employed for some investigated devices to improve its breakdown characteristics. An air-bridge technology using an electroplated $\mathrm{Au}$ is used to connect the source pads of multifinger devices.

\section{Small-signal modeling}

In bottom-up modeling technique, a multibias small-signal measurement is carried out over a range of bias points, and a large-signal model is then determined from the small-signal model derived at each of these bias points. Therefore, the accuracy of the constructed largesignal model depends on the accuracy of the bias-dependent small-signal model, which should reflect the electrical and physical characteristics of the device. Accurate determination of the intrinsic bias-dependent circuit of GaN HEMT small-signal model requires an efficient extraction method for the parasitic elements of the device. In (Jarndal \& Kompa, 2005), an efficient reliable model parameter extraction method, applied for GaN HEMT, was developed. This method uses only a cold S-parameter measurement for accurate determination of the parasitic elements. The main advantage of this method is that it gives reliable values for the parasitic elements of the device without need for additional measurements or separate test patterns. Since the knowledge of distributed effects is important to identify the device parasitic elements for further minimization, a 22-element distributed model shown in Figure 2 is used as a small-signal model for GaN HEMT. This model is general and applicable for large gate periphery devices. The main advantages of this model are as follows.

- It accounts for all expected parasitic elements of the device.

- It reflects the physics of the device over a wide bias and frequency range.

Therefore, this model can be suitable for scalable large-signal model construction.

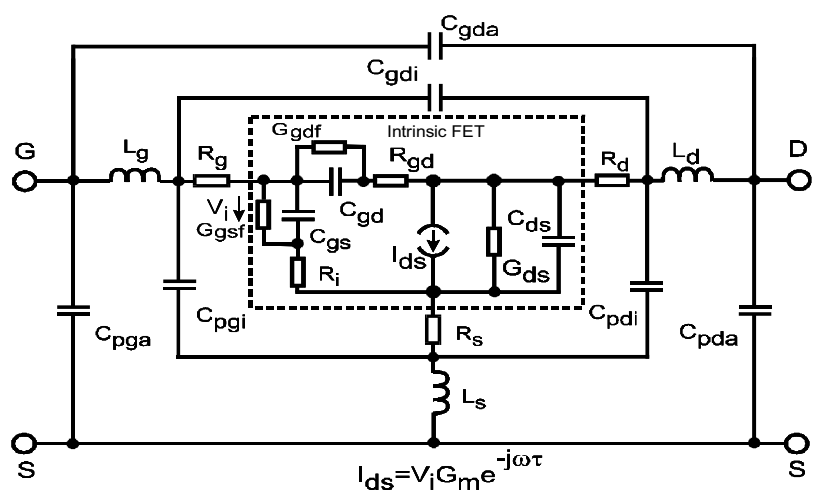

Fig. 2. 22-element distributed small-signal model for active GaN HEMT. 


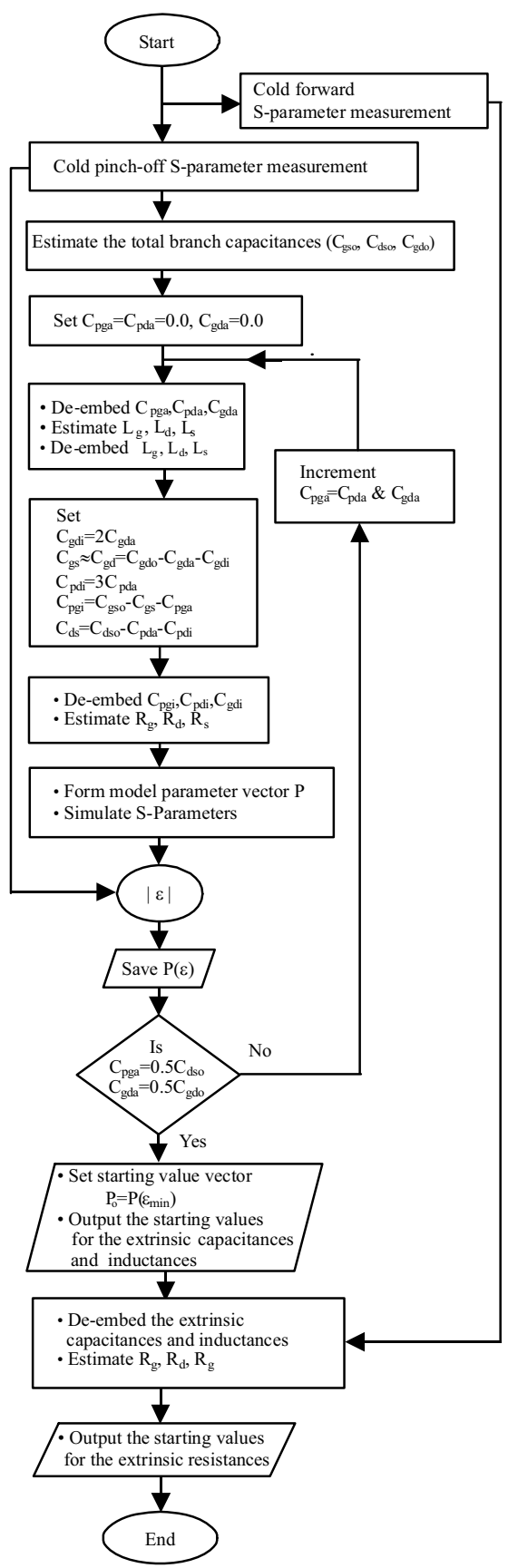

Fig. 3. Flowchart of the small-signal model parameter starting value generation algorithm. (c) 2005 IEEE. Reprinted with permission. 
In the extrinsic part of this model, $\mathrm{C}_{\mathrm{pga}}, \mathrm{C}_{\mathrm{pda}}$ and $\mathrm{C}_{\text {gda }}$ account for parasitic elements due to the pad connections, measurement equipment, probes, and probe tip-to-device contact transitions; while $\mathrm{C}_{\mathrm{pgi}}, \mathrm{C}_{\mathrm{pdi}}$, and $\mathrm{C}_{\mathrm{gdi}}$ account for interelectrode and crossover capacitances (due to air-bridge source connections) between gate, source, and drain. $R_{g}, R_{d}$, and $R_{s}$ represent contact and semiconductor bulk resistances; while $\mathrm{L}_{\mathrm{g}}, \mathrm{L}_{\mathrm{d}}$, and $\mathrm{L}_{\mathrm{s}}$ model effect of metallization inductances. In the intrinsic part, charging and discharging process for depletion region under the gate is described by $C_{g s}, R_{i}, C_{g d}$ and $R_{g d}$. The gate forward and breakdown conductions are represented by $G_{g s f}$ and $G_{g d f}$, respectively. Variation of the channel conduction with remote gate voltage is described by $\mathrm{G}_{\mathrm{m}}$; while the channel conductance controlled by local drain voltage is represented by $G_{d s}$. $C_{d s}$ model the capacitance between the drain and source electrodes separated by the depletion region in electrostatic sense. Transit time of electrons in the channel at high-speed input signal is described by $\tau$.

\subsection{Extrinsic parameter extraction}

Many of the model parameters in Figure 2 are difficult if not impossible to determine directly from measurements. Therefore, these parameters are determined through an optimization algorithm. The efficiency of this algorithm depends on the quality of starting values and the number of optimization variables. Under cold pinch-off condition, the equivalent circuit in Figure 2 can be simplified by excluding some elements, thereby reducing the number of unknowns. For further minimization of the number of optimization variables, only the extrinsic elements of the model will be optimized, while the intrinsic elements are determined from the deembedded Y-parameters. Under this bias condition, the reactive elements of the model are strongly correlated (Jarndal \& Kompa, 2005). Therefore, the starting values estimation can be carried out in a way that takes this correlation into account. In addition, the S-parameter measurements must cover the frequency range where this correlation is more obvious. The required measurements frequency range for reliable starting values generation reduces for larger devices, e.g., up to $20 \mathrm{GHz}$ for an $8 \times 125-\mu \mathrm{m}$ device. The proposed technique for starting values generation is based on searching for the optimal distribution of the total capacitances. This is achieved by scanning the outer capacitance values within the specified ranges. For each scanned value, the interelectrode capacitances are assigned suitable values and then deembedded from the measured Yparameters. The rest of the model parameters are then estimated from the stripped Yparameters. The whole estimated parameters are then used to simulate the device Sparameters, which are then compared with the measured ones. Using this systematic searching procedure, high-quality measurement-correlated starting values for the smallsignal model parameters can be found. The closeness of the starting values to the real values simplifies the next step of parameters optimization since the risk of a local minimum is minimized.

\section{A. Generation of starting value of small-signal model parameters}

The starting values generation procedure is described by the flowchart in Figure 3. As shown in this flowchart, the starting values of the extrinsic capacitances and inductances are generated from pinch-off measurements, while those of extrinsic resistances are generated 
from forward measurements. The whole starting values generating procedure can be summarized as follows.

Step 1) Let $V_{G S}<-V_{\text {pinch-off }}$ and $V_{D S}=0.0 \mathrm{~V}$. In this case, the equivalent circuit in Figure 2 of the active device can be used for this cold pinch-off device if the drain current source and the output channel conductance are excluded. Moreover, at low frequencies (in the megahertz range), this circuit can be reduced to a capacitive network shown in Figure 4 and the Y-parameters of this equivalent circuit can be written as

$$
\begin{aligned}
& Y_{11}=j \omega\left(C_{g s o}+C_{g d o}\right) \\
& Y_{22}=j \omega\left(C_{d s o}+C_{g d o}\right) \\
& Y_{12}=Y_{21}=-j \omega C_{g d o}
\end{aligned}
$$

where

$$
\begin{gathered}
C_{g d o}=C_{g d a}+C_{g d i}+C_{g d} \\
C_{g s o}=C_{p g a}+C_{p g i}+C_{g s} \\
C_{d s o}=C_{p d a}+C_{p d i}+C_{d s} .
\end{gathered}
$$

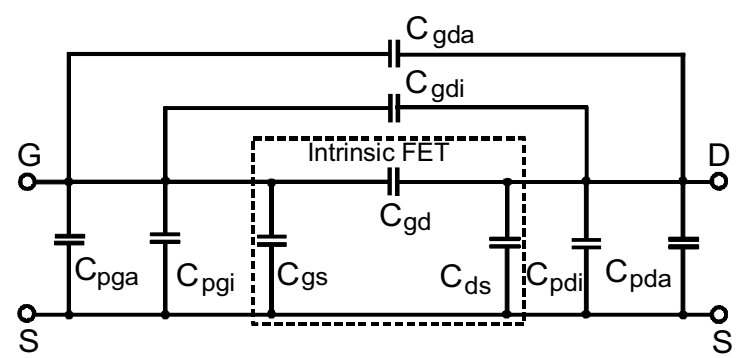

Fig. 4. Equivalent circuit model for cold pinch-off GaN HEMT at low frequency.

The total capacitances for gate-source, gate-drain, and drain-source branches are determined from the low frequency range of pinch-off S-parameter measurements, which are converted to Y-parameter.

Step 2) The next step is searching for the optimal distribution of the total capacitances, which gives the minimum error between the measured and simulated $\mathrm{S}$ parameters. This is achieved by scanning $\mathrm{C}_{\mathrm{pga}}, \mathrm{C}_{\mathrm{pda}}$, and $\mathrm{C}_{\mathrm{gda}}$ values within the specified ranges. $C_{\mathrm{pga}}$ and $\mathrm{C}_{\mathrm{pda}}$ are scanned from 0 to $0.5 \mathrm{C}_{\mathrm{dso}}$ while $\mathrm{C}_{\mathrm{gda}}$ is scanned from 0 to $0.5 \mathrm{C}_{\text {gdo }}$. During the scanning process, $C_{\mathrm{pga}}$ is assumed to be equal to $\mathrm{C}_{\mathrm{pda}}$

$$
C_{p g a} \cong C_{p d a} \text {. }
$$

The gate-drain interelectrode capacitance $C_{\text {gdi }}$ is assumed to be twice the $C_{\text {gda }}$ pad capacitance value

$$
C_{g d i} \cong 2 C_{g d a} .
$$

For symmetrical gate-source and gate-drain spacing, the depletion region will be uniform under pinch-off, so that 


$$
C_{g s} \cong C_{g d}=C_{g d o}-C_{g d i}-C_{g d a} .
$$

The value of $C_{\text {pgi }}$ is calculated using

$$
C_{p g i}=C_{g s o}-C_{g s}-C_{p g a} .
$$

With the GaN devices under analysis, $\mathrm{C}_{\mathrm{pdi}}$ is a significant part of the total drainsource capacitance. Therefore, it is found that the assumption

$$
C_{p d i} \cong 3 C_{p d a}
$$

minimizes the error between the simulated and measured S-parameters. For medium and high frequency range, the intrinsic transistor of the pinch-off model is represented in T-network as shown in Figure 5 where the interelectrode capacitances $\left(\mathrm{C}_{\mathrm{pgi}}, \mathrm{C}_{\mathrm{pdi}}\right.$, and $\mathrm{C}_{\mathrm{gdi}}$ ) have been absorbed in the intrinsic capacitances $\left(C_{g s}, C_{d s}\right.$, and $\left.C_{g d}\right)$. The values for $C_{p g a}, C_{p d a}$, and $C_{g d a}$ are deembedded from $Y-$ parameter and then converted to Z-parameter.

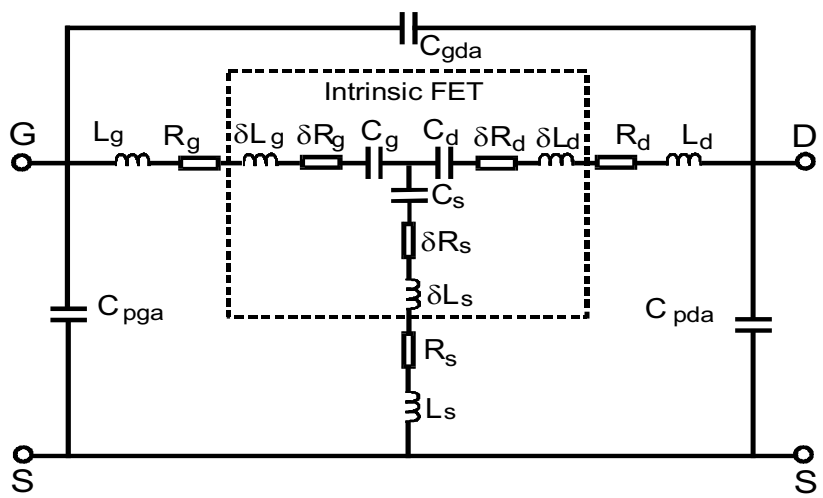

Fig. 5. Equivalent circuit model for cold pinch-off GaN HEMT at medium and high frequency.

This stripped Z-parameter can be written as

$$
\begin{array}{r}
Z_{11}=R_{g}+R_{s}+j \omega\left(L_{g}+L_{s}\right)+\frac{1}{j \omega}\left(\frac{1}{C_{g}}+\frac{1}{C_{s}}\right)+\delta Z_{g} \\
Z_{22}=R_{d}+R_{s}+j \omega\left(L_{d}+L_{s}\right)+\frac{1}{j \omega}\left(\frac{1}{C_{d}}+\frac{1}{C_{s}}\right)+\delta Z_{d} \\
Z_{12}=Z_{21}=R_{s}+j \omega L_{s}+\frac{1}{j \omega C_{s}}+\delta Z_{s}
\end{array}
$$

where

$$
\begin{aligned}
& \delta Z_{g}=\delta R_{g}+\delta R_{s}+j \omega\left(\delta L_{g}+\delta L_{s}\right) \\
& \delta Z_{d}=\delta R_{d}+\delta R_{s}+j \omega\left(\delta L_{d}+\delta L_{s}\right)
\end{aligned}
$$




$$
\delta Z_{s}=\delta R_{s}+j \omega \delta L_{s} .
$$

$\delta Z_{g}, \delta Z_{d}$, and $\delta Z_{s}$ represent correction terms related to the intrinsic parameters of the model. Ignoring the correction terms and multiplying the Z-parameters by $\omega$ and then taking the imaginary parts gives

$$
\begin{array}{r}
\operatorname{Im}\left[\omega Z_{11}\right]=\left(L_{g}+L_{s}\right) \omega^{2}-\left(\frac{1}{C_{g}}+\frac{1}{C_{s}}\right) \\
\operatorname{Im}\left[\omega Z_{22}\right]=\left(L_{d}+L_{s}\right) \omega^{2}-\left(\frac{1}{C_{d}}+\frac{1}{C_{s}}\right) \\
\operatorname{Im}\left[\omega Z_{12}\right]=L_{s} \omega^{2}-\frac{1}{C_{s}} .
\end{array}
$$

Hence, the values of $L_{g}, L_{d}$, and $L_{s}$ can be extracted from the slope of $\operatorname{Im}\left[\omega Z_{i j}\right]$ versus $\omega^{2}$ curve. The estimated values of the inductances described above and the interelectrode capacitances $\left(\mathrm{C}_{\mathrm{pgi}}, \mathrm{C}_{\mathrm{pdi}}\right.$, and $\left.\mathrm{C}_{\mathrm{gdi}}\right)$ are deembedded. However, the incomplete deembedding of the outer capacitances and the inductances introduce nonlinear frequency dependence in the real part of deembedded Z-parameters. By multiplying the deembedded Z-parameter by $\omega^{2}$, this effect is reduced (Jarndal \& Kompa, 2005). Ignoring the correction terms and multiplying the deembedded Zparameter by $\omega^{2}$ and then taking the real part of this $\mathrm{Z}$-parameter gives

$$
\begin{array}{r}
\operatorname{Re}\left[\omega^{2} Z_{11}\right]=\omega^{2}\left(R_{g}+R_{s}\right) \\
\operatorname{Re}\left[\omega^{2} Z_{22}\right]=\omega^{2}\left(R_{d}+R_{s}\right) \\
\operatorname{Re}\left[\omega^{2} Z_{12}\right]=\omega^{2} R_{s} .
\end{array}
$$

By linear regression, the value of $R_{g}+R_{s}, R_{d}+R_{s}$, and $R_{s}$ can be extracted from the slope of $\operatorname{Re}\left[\omega^{2} Z_{\mathrm{ij}}\right]$ versus $\omega^{2}$ curves. The resulting estimated parameters are used to simulate the device S-parameters, which are then compared with the measured ones to calculate the residual fitting error $(\varepsilon)$. The outer capacitances $\left(\mathrm{C}_{\mathrm{pga}}, \mathrm{C}_{\mathrm{pda}}\right.$, and $\left.\mathrm{C}_{\mathrm{gda}}\right)$ are incremented, and the procedure is repeated until $\mathrm{C}_{\mathrm{pga}}\left(\mathrm{C}_{\mathrm{pda}}\right)$ is equal to $0.5 \mathrm{C}_{\mathrm{dso}}$ and $\mathrm{C}_{\mathrm{gda}}$ is equal to $0.5 \mathrm{C}_{\mathrm{gdo}}$. The vector of model parameters $P\left(\varepsilon_{\mathrm{min}}\right)$, corresponding to the lowest error $\varepsilon_{\min }$, is then taken as the appropriate starting value.

Step 3) Because of unavoidable high measurement uncertainty for cold pinch-off device, the determination of a reliable starting value for the extrinsic resistances is difficult if not impossible. More reliable starting value was generated using cold gate forward S-parameter measurements at high gate voltage greater than or equal to $2 \mathrm{~V}$. This is due to the higher conduction band of GaN-based HEMT with respect to the corresponding GaAs-based HEMT. Therefore, significantly higher voltages have to be applied to reach the condition when the influence of the gate capacitance is negligible. The determined values of extrinsic capacitances and inductances, in Step 2), are deembedded from the gate-forward measurements. The starting values of the extrinsic resistances are then estimated from the stripped forward Zparameters. 


\section{B. Model parameter optimization}

The procedure for the generation of starting values of the model parameters was discussed in Section 3.1-A. Here, the result of the optimal value for each model parameter is presented. Model parameters optimization has been done based on the principle of bidirectional optimization technique proposed by Lin and Kompa (Lin \& Kompa, 1994). This technique works successfully for lumped small-signal model, but cannot be used efficiently for distributed model. This is due to the extra parasitic elements of this model, which increase the searching space. Now, this algorithm can be modified to become applicable for the distributed model, where the closeness of the generated starting value to the true value allows the searching space to be reduced by optimizing only the extrinsic parameters. At each iteration through the optimization process, the extrinsic parameters are assigned suitable values and then deembedded from the measured data to determine the intrinsic Yparameters. The intrinsic model parameters are then estimated by means of data fitting from the deembedded measurements. The whole estimated model parameters are then used to fit the measured S-parameters. This process is continued to find the optimal model parameters. In this case, the optimization problem is a nonlinear multidimensional one, whose objective function is likely to have multiple local minima. Furthermore, the cold pinch-off device measurements have a high uncertainty (HP8510B network analyzer manual, 1987). These two factors increase the probability of trapping into a local minimum, which requires a careful formulation for the objective function to avoid the local minimum problem. The magnitude of the error between the measured S-parameter and its simulated value can be expressed as (Jarndal \& Kompa, 2005)

$$
\varepsilon_{i j}=\frac{\left|\operatorname{Re}\left(\delta S_{i j, n}\right)\right|+\left|\operatorname{Im}\left(\delta S_{i j, n}\right)\right|}{W_{i j}} \quad, \mathrm{i}, \mathrm{j}=1,2 ; \mathrm{n}=1,2, \ldots, \mathrm{N}
$$

where

$$
\begin{gathered}
W_{i j}=\max \left[\left|S_{i j}\right|\right] \quad, \mathrm{i}, \mathrm{j}=1,2 ; \mathrm{i} \neq \mathrm{j} \\
W_{i i}=1+\left|S_{i i}\right| \quad, \mathrm{i}=1,2
\end{gathered}
$$

and $N$ is the total number of data points. $\delta S$ is the difference between the measured Sparameter coefficient and its simulated value. The weighting factor $(W)$ deemphasizes data region with higher reflection coefficients due to the involved higher measurement uncertainty. The scalar error is then expressed as

$$
\varepsilon_{s}=\frac{1}{N} \sum_{n=1}^{N}\|\|^{*}\left(f_{n}\right) \|_{1}
$$

where

$$
{ }^{*}\left(f_{n}\right)=\left[\begin{array}{ll}
\varepsilon_{11}\left(f_{n}\right) & \varepsilon_{12}\left(f_{n}\right) \\
\varepsilon_{21}\left(f_{n}\right) & \varepsilon_{22}\left(f_{n}\right)
\end{array}\right]
$$

defined at each frequency point. However, the objective function that is based on Sparameters alone to minimize the fitting error may not necessarily lead to physically relevant values of the model parameters (Kompa \& Novotny, 1997). For further 
enhancement of the objective function, another performance quantity, depending on the final application, will be considered. The main application of GaN-based HEMT is power amplifier design. For power amplifier design, the output and input impedance, the device gain, and stability factor are important for the design of matching networks. These factors can be expressed as a function of S-parameters and fitted during the optimization. The stability factor defined at the output plane of the device at each frequency can be expressed as

$$
K=\frac{1-\left|S_{22}\right|^{2}}{\left|S_{22}-S_{11}^{*} \Delta_{s}\right|+\left|S_{12} S_{21}\right|}
$$

where $S^{*}$ is the complex conjugate and $\Delta_{\mathrm{S}}$ is the determinant of S-parameter matrix at each frequency (Edwards \& Sinsky, 1992). The fitting error of the stability factor is given by

$$
\varepsilon_{K}=\frac{1}{N} \sum_{k=1}^{N}\left|K_{\text {meas }}-K_{\text {sim }}\right|
$$

where $K_{\text {meas }}$ and $K_{\text {sim }}$ are the stability factors from the measured and simulated S-parameters, respectively. With regard to the device gain, the maximally efficient gain defined in (Kotzebue,1976) is a more suitable one, since it remains finite even for an unstable device. This gain may be defined at each frequency as

$$
G=\frac{\left|S_{21}\right|^{2}-1}{\ln \left|S_{21}\right|^{2}} .
$$

The error in the gain may thus, be expressed as

$$
\varepsilon_{G}=\frac{1}{N} \sum_{m=1}^{N}\left|G_{\text {meas }}-G_{\text {sim }}\right|
$$

where $G_{\text {meas }}$ and $G_{\text {sim }}$ are the gains computed from the measured and modeled S-parameters. The fitting error can be defined in terms of the three error components as

$$
\varepsilon=\sqrt{\frac{1}{3}\left(\varepsilon_{s}{ }^{2}+\varepsilon_{K}{ }^{2}+\varepsilon_{G}{ }^{2}\right)} .
$$

The modified Simplex optimization algorithm proposed in (Kompa \& Novotny, 1997) is used to minimize the objective function in (33).

The extraction procedure was applied to different GaN HEMT sizes. Table 1 presents the final optimised results for extrinsic parameters extraction. As it can be observed in the table, the extracted pad capacitances $\left(\mathrm{C}_{\mathrm{pga}}, \mathrm{C}_{\mathrm{pda}}\right.$, and $\left.\mathrm{C}_{\mathrm{gda}}\right)$ are in proportion with the gate width. There is no significant difference between the pad capacitances of $8 \times 125-\mu \mathrm{m}$ and $8 \times 250-\mu \mathrm{m}$ devices because the pad connection area is related mainly to the number of fingers. The inter-electrode capacitances $\left(\mathrm{C}_{\mathrm{pdi}}\right.$ and $\left.\mathrm{C}_{\mathrm{gdi}}\right)$ are also in proportion with the gate width. Due to the small values of $R_{g}$ and $R_{s}$, for larger devices, $C_{p g i}$ cannot be separated completely from the intrinsic capacitance $C_{g s}$. However, the sum of $C_{p g i}$ and $C_{g s}$ is in proportion with the gate width. By direct scaling of the $8 \times 250-\mu \mathrm{m}$ device, the expected values of $\mathrm{C}_{\mathrm{gda}}$ and $\mathrm{C}_{\mathrm{gdi}}$ for 
$8 \times 125-\mu \mathrm{m}$ device are $20 \mathrm{fF}$ and $40 \mathrm{fF}$, respectively. Due to the smaller values of these elements and also due to the smaller values of $\mathrm{Lg}_{g}$ and $\mathrm{L}_{\mathrm{d}}$ for this device, $\mathrm{C}_{\mathrm{gda}}$ and $\mathrm{C}_{\text {gdi }}$ cannot be separated form $C_{g d}$. The parasitic inductance includes the self-inductance due the metallization contact and the mutual inductance between the metal interconnection. The mutual inductance increases by increasing the number of fingers. For this reason, there is a considerable increase of $L_{d}$ and $L_{g}$ values for $16 \times 250-\mu \mathrm{m}$ device with respect to $8 \times 125-\mu \mathrm{m}$ device (Jarndala \& Kompa, 2006). The parasitic resistances $\left(R_{d}\right.$ and $\left.R_{s}\right)$ are inversely proportional with the gate width. However, this is not the case with $R_{g}$, which is proportional with the unite-gate-width and inversely proportional with the number of gate fingers as reported in (Goyal et al., 1989).

\begin{tabular}{|l|l|l|l|l|}
\hline Parameter & $\mathrm{W}_{\mathrm{g}}=16 \times 250 \mu \mathrm{m}$ & $\mathrm{W}_{\mathrm{g}}=8 \times 250 \mu \mathrm{m}$ & $\mathrm{W}_{\mathrm{g}}=8 \times 125 \mu \mathrm{m}$ & $\mathrm{W}_{\mathrm{g}}=2 \times 50 \mu \mathrm{m}$ \\
\hline $\mathrm{C}_{\text {pga }}(\mathrm{fF})$ & 233.5 & 89.8 & 86.9 & 9.97 \\
$\mathrm{C}_{\text {pgi }}(\mathrm{fF})$ & 39.6 & 234.8 & 332.2 & 7.09 \\
$\mathrm{C}_{\mathrm{gs}}(\mathrm{fF})$ & 1508.4 & 538.6 & 255.8 & 15.38 \\
\hline $\mathrm{C}_{\text {gda }}(\mathrm{fF})$ & 121.6 & 41.7 & 0.0 & 0.47 \\
$\mathrm{C}_{\text {gdi }}(\mathrm{fF})$ & 265.6 & 96.5 & 0.0 & 0.86 \\
$\mathrm{C}_{\text {gd }}(\mathrm{fF})$ & 1285.7 & 757.8 & 517.4 & 20.17 \\
\hline $\mathrm{C}_{\text {pda }}(\mathrm{fF})$ & 206.4 & 90.9 & 86.3 & 7.13 \\
$\mathrm{C}_{\text {pdi }}(\mathrm{fF})$ & 790.7 & 390.2 & 245 & 29.42 \\
$\mathrm{C}_{\mathrm{ds}}(\mathrm{fF})$ & 0.0 & 0.0 & 1.0 & 0.0 \\
\hline $\mathrm{L}_{\mathrm{g}}(\mathrm{pH})$ & 122.3 & 81.9 & 57.3 & 46.55 \\
$\mathrm{~L}_{\mathrm{d}}(\mathrm{pH})$ & 110.9 & 75.4 & 54.5 & 47.9 \\
$\mathrm{~L}_{\mathrm{s}}(\mathrm{pH})$ & 3.6 & 5.7 & 5.6 & 6.25 \\
\hline $\mathrm{R}_{\mathrm{g}}(\Omega)$ & 1.1241 & 2.8 & 1.7 & 4.8 \\
$\mathrm{R}_{\mathrm{d}}(\Omega)$ & 0.71424 & 1.4 & 2.3 & 11.8 \\
$\mathrm{R}_{\mathrm{s}}(\Omega)$ & 0.25152 & 0.5 & 0.9 & 5.47 \\
\hline $\mathrm{R}_{\mathrm{i}}(\Omega)$ & 0.0 & 0.0 & 0.0 & 0.0 \\
$\mathrm{R}_{\mathrm{gd}}(\Omega)$ & 0.1 & 0.0 & 0.0 & 0.0 \\
\hline $\mathrm{G}_{\mathrm{m}}(\mathrm{mS})$ & 0.0 & 0.0 & 0.0 & 0.0 \\
$\mathrm{\tau}(\mathrm{ps})$ & 0.0 & 3.3 & 0.0 & 0.0 \\
$\mathrm{G}_{\mathrm{ds}}(\mathrm{mS})$ & 0.34 & 0.0 & 0.26 & 0.0 \\
\hline $\mathrm{G}_{\mathrm{gsf}}(\mathrm{mS})$ & 2.3 & 0.6 & 0.4 & 0.0 \\
$\mathrm{G}_{\text {gdf }}(\mathrm{mS})$ & 0.24 & 0.25 & 0.2 & 0.0 \\
\hline
\end{tabular}

Table 1. Extracted model parameters for different GaN HEMT sizes under cold pinch-off bias condition ( $\mathrm{V}_{\mathrm{DS}}=0 \mathrm{~V}$ and $\mathrm{V}_{\mathrm{GS}}=\mathrm{V}_{\text {pinch-off }}$ ). (C) 2006 IEEE. Reprinted with permission.

\subsection{Intrinsic parameter extraction}

After deembedding the extracted extrinsic parameters in Section 3.1, the bias-dependent intrinsic parameters can be extracted. An efficient technique is developed for extracting of the optimal value of the intrinsic element. In this technique, the intrinsic $Y$-parameters are formulated in a way where the optimal intrinsic element value can be extracted using simple linear data fitting (Jarndal \& Kompa, 2005). The admittance for the intrinsic gatesource branch $Y_{g s}$ is given by 


$$
Y_{g s}=Y_{i, 11}+Y_{i, 12}=\frac{G_{g s f}+j \omega C_{g s}}{1+R_{i} G_{g s f}+j \omega R_{i} C_{g s}} .
$$

By defining a new variable $D$ as

$$
D=\frac{\left|Y_{g s}\right|^{2}}{\operatorname{Im}\left[Y_{g s}\right]}=\frac{G_{g s f}^{2}}{\omega C_{g s}}+\omega C_{g s} .
$$

$C_{g s}$ can be determined from the slope of the curve for $\omega D$ versus $\omega^{2}$ by linear fitting, where $\omega$ is the angular frequency. By redefining $D$ as

$$
D=\frac{Y_{g s}}{\operatorname{Im}\left[Y_{g s}\right]}=\frac{G_{g s f}\left(1+R_{i} G_{g s f}\right)}{\omega C_{g s}}+\omega R_{i} C_{g s}-j .
$$

$\mathrm{R}_{\mathrm{i}}$ can be determined from the plot of the real part of $\omega D$ versus $\omega^{2}$ by linear fitting. $G_{g s f}$ can be determined from the real part of $Y_{g s}$ at low frequencies (in the megahertz range). The admittance for the intrinsic gate-drain branch $Y_{g d}$ is given by

$$
Y_{g d}=-Y_{i, 12}=\frac{G_{g d f}+j \omega C_{g d}}{1+R_{g d} G_{g d f}+j \omega R_{g d} C_{g d}} .
$$

The same procedure, given in (35) and (36), can be used for extracting $C_{g d}, R_{g d}$, and $G_{g d f}$. The admittance of the intrinsic transconductance branch $Y_{g m}$ can be expressed as

$$
Y_{g m}=Y_{i, 21}-Y_{i, 12}=\frac{G_{m} e^{-j \omega \tau}}{1+R_{i} G_{g s f}+j \omega C_{g s}} .
$$

By redefining $D$ as

$$
D=\left|\frac{Y_{g s}}{Y_{g m}}\right|^{2}=\left(\frac{G_{g s f}}{G_{m}}\right)^{2}+\left(\frac{C_{g s}}{G_{m}}\right)^{2} \omega^{2} .
$$

$G_{m}$ can be determined from the slope of the curve for $D$ versus $\omega^{2}$ by linear fitting. By redefining $D$ as

$$
D=\left(G_{g s f}+j \omega C_{g s}\right) \frac{Y_{g m}}{Y_{g s}}=G_{m} e^{-j \omega \tau}
$$



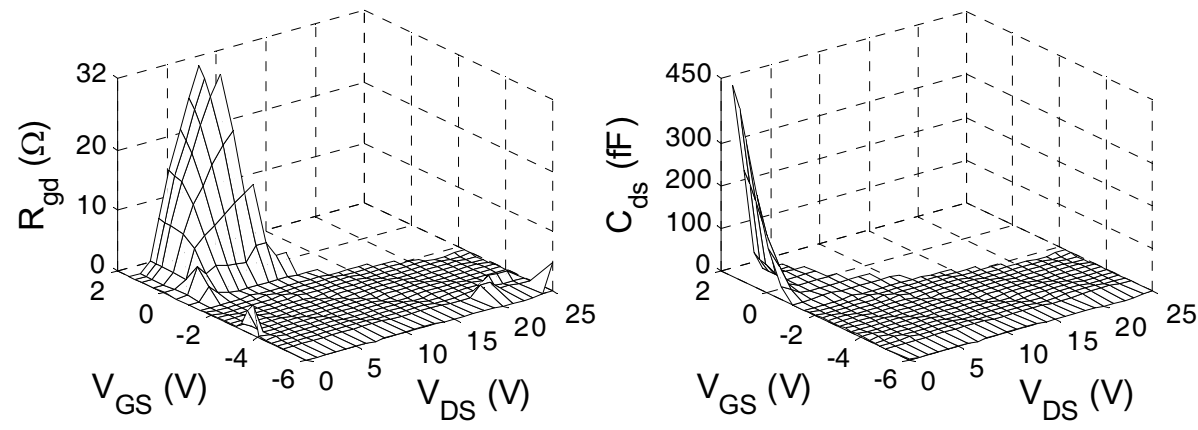

Fig. 6. Extracted $R_{g d}$ and $C_{d s}$ as a function of the extrinsic voltages for a GaN HEMT with a $2 \times 50-\mu \mathrm{m}$ gate width. (C) 2005 IEEE. Reprinted with permission.
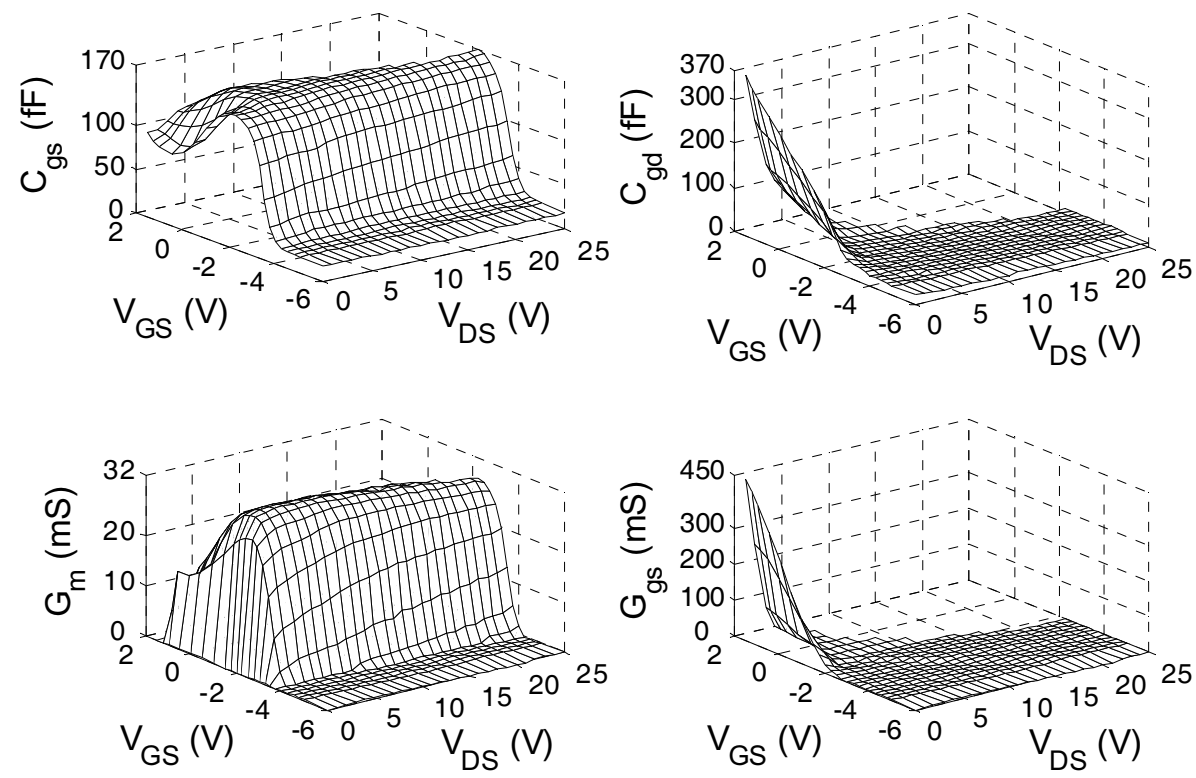

Fig. 7. Extracted $C_{g s}, C_{g d}, G_{m}$, and $G_{d s}$ as a function of the extrinsic voltages for a GaN HEMT with a $2 \times 50-\mu \mathrm{m}$ gate width. (C) 2005 IEEE. Reprinted with permission. 

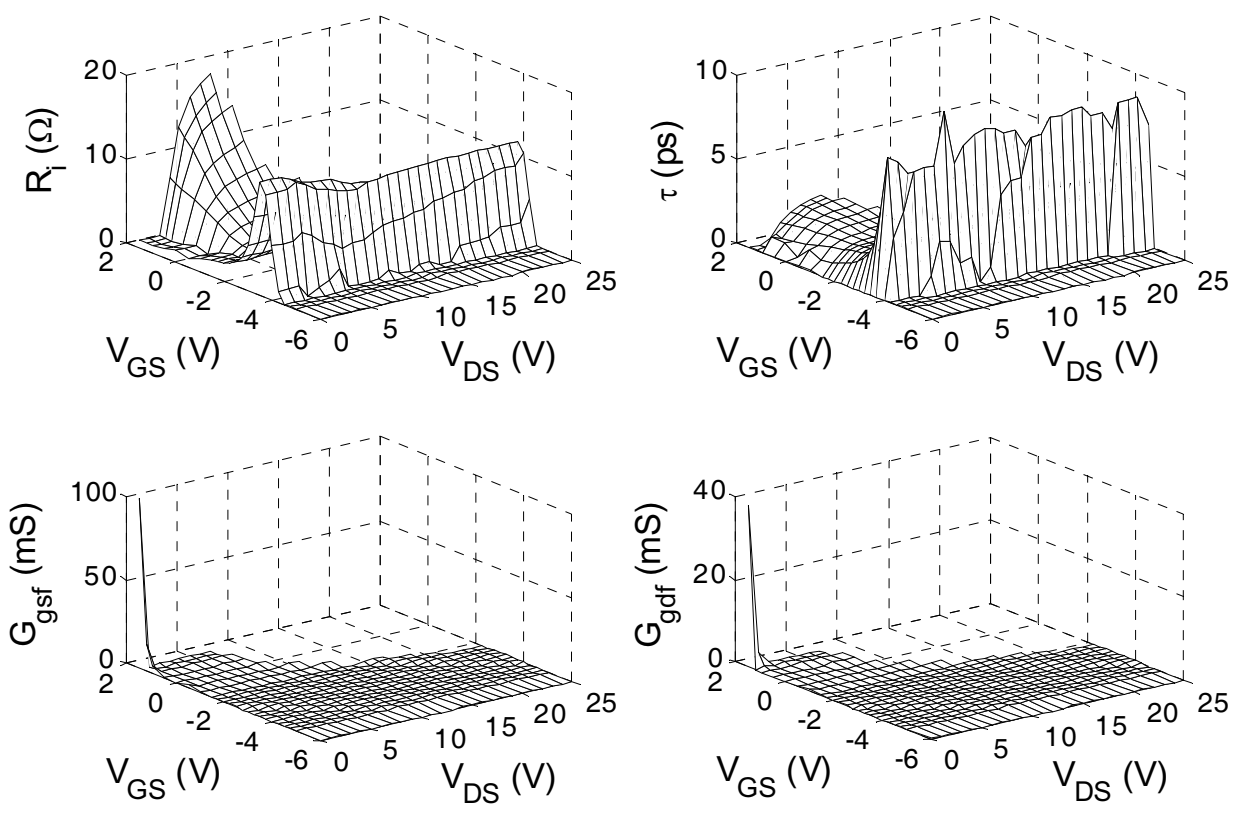

Fig. 8. Extracted $R_{i}, \tau, G_{g s f}$, and $G_{g d f}$ as a function of the extrinsic voltages for a GaN HEMT with a $2 \times 50-\mu \mathrm{m}$ gate width. (c) 2005 IEEE. Reprinted with permission.

$\tau$ can be determined from the plot of the phase of $D$ versus $\omega$ by linear fitting. The admittance of the intrinsic drain-source branch $Y_{d s}$ can be expressed as

$$
Y_{d s}=Y_{i, 22}+Y_{i, 12}=G_{d s}+j \omega C_{d s} .
$$

$C_{d s}$ can be extracted from the plot of the imaginary part of $Y_{d s}$ versus $\omega$ by linear fitting. Due to the frequency-dependent effect in the output conductance $G_{d s}$, its value is determined from the curve of $\omega \operatorname{Re}\left[Y_{d s}\right]$ versus $\omega$ by linear fitting.

Figs. 6-8 present extracted intrinsic parameters for GaN HEMT using the proposed procedure under different extrinsic bias voltages. The extraction results show the typical expected characteristics of GaN HEMT. The reliability of the extraction results was demonstrated in (Jarndal \& Kompa, 2005) in terms of the reverse modeling of the effective gate length for the same analysed devices. The accuracy of the proposed small signal modeling approach is verified through S-parameter simulation for different device sizes under different bias conditions. As it can be seen in Figs. 9 and 10, the model can simulate the S-parameter accurately. Also it can predict the kink effect in $S_{22}$, which occurs in larger size FETs (Lu et al., 2001). 


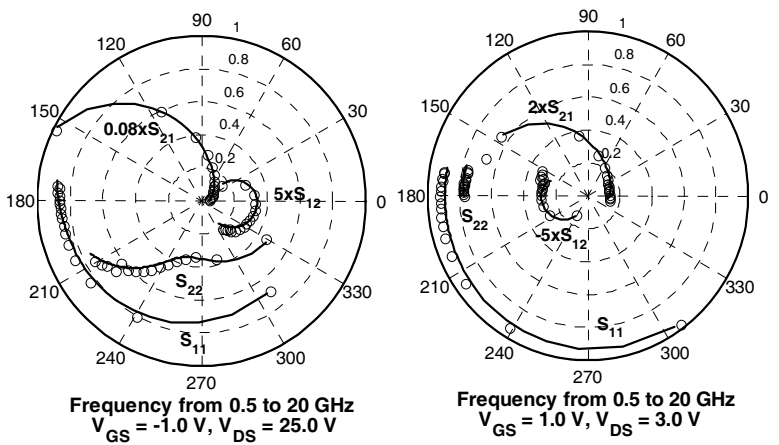

Fig. 9. Comparison of measured S-parameters of a 8x125- $\mu \mathrm{m}$ GaN HEMT (circles) with simulation results (lines) at $\left(\mathrm{V}_{\mathrm{GS}}=-1, \mathrm{~V}_{\mathrm{DS}}=25 \mathrm{~V}\right)$ and $\left(\mathrm{V}_{\mathrm{GS}}=1 \mathrm{~V}, \mathrm{~V}_{\mathrm{DS}}=3 \mathrm{~V}\right)$. (c) 2006 IEEE. Reprinted with permission.
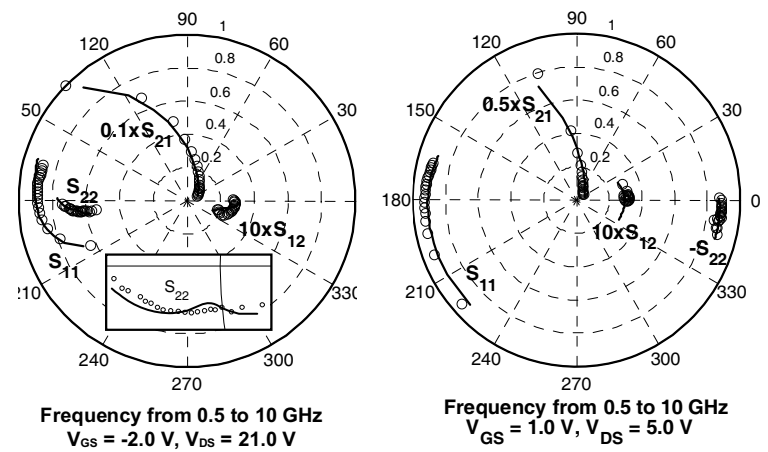

Fig. 10. Comparison of measured S-parameters of a 16x250- $\mu \mathrm{m}$ GaN HEMT (circles) with simulation results (lines) at $\left(\mathrm{V}_{\mathrm{GS}}=-2, \mathrm{~V}_{\mathrm{DS}}=21 \mathrm{~V}\right)$ and $\left(\mathrm{V}_{\mathrm{GS}}=1 \mathrm{~V}, \mathrm{~V}_{\mathrm{DS}}=5 \mathrm{~V}\right)$. (c) 2006 IEEE. Reprinted with permission.

\section{Large-signal modeling}

Under RF large-signal operation, the values of the intrinsic-elements of the GaN HEMT model in Figure 2 vary with time and become dependent on the terminal voltages. Therefore the intrinsic part of this model can be described by the equivalent-circuit model shown in Figure 11. In this circuit, two quasi-static gate-current sources $I_{g s}$ and $I_{g d}$ and two quasi-static gate-charge sources $Q_{g s}$ and $Q_{g d}$ are used to describe the conduction and displacement currents. The nonquasi-static effect in the channel charge is approximately modeled with two bias-dependent resistors $R_{i}$ and $R_{g d}$ in series with $Q_{g s}$ and $Q_{g d}$, respectively. This implementation is simpler and it improves the accuracy of the model up to millimeter-wave frequencies (Schmale \& Kompa, 1997). A nonquasistatic drain-current model which accounts for trapping and self-heating effects is embedded in the proposed large-signal model. The drain-current value is determined by the applied intrinsic voltages $V_{g s}$ and $V_{d s}$, whereas the amount of trapping induced current dispersion is controlled by the ac components of these voltages. These components are extracted from the intrinsic voltage 
using RC high-pass circuits at gate and drain sides, as shown in Figure 11. The capacitors $\mathrm{C}_{\mathrm{GT}}$ and $\mathrm{C}_{\mathrm{DT}}$ values are selected to be $1 \mathrm{pF}$ to provide a "macroscopic" modeling of charges stored in the surface and buffer traps. These charges are almost related to the leakage currents from the gate metal edge to the surface (Vetury et al., 2001) or from the channel into the buffer layer (Kohn et al., 2003). The small leakage currents in the gate and drain paths are realized with large (on the order of $1 \mathrm{M} \Omega$ ) resistances $R_{\mathrm{GT}}$ and $\mathrm{R}_{\mathrm{DT}}$ in series with $\mathrm{C}_{\mathrm{GT}}$ and $\mathrm{C}_{\mathrm{DT}}$, respectively.

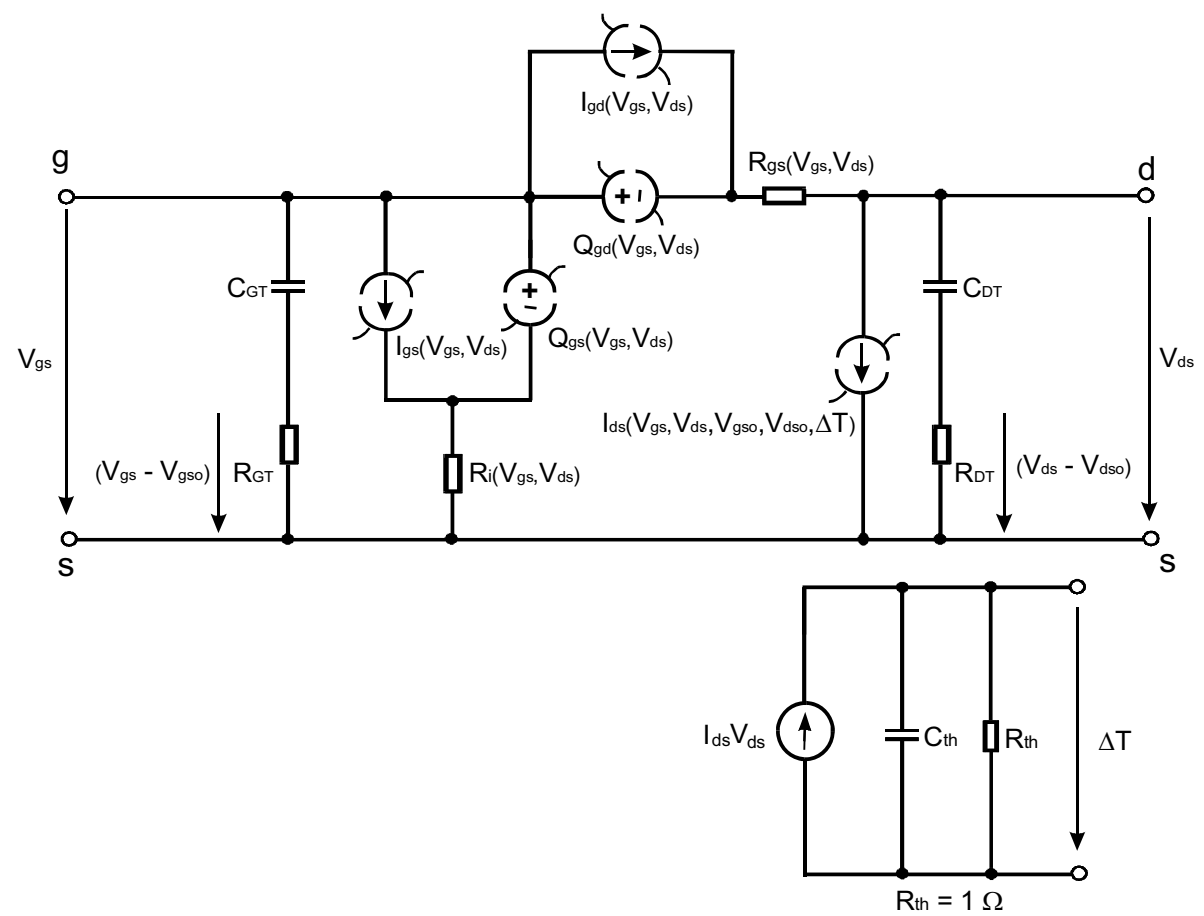

Fig. 11. Large-signal model for GaN HEMT including self-heating and trapping effects.

This implementation makes the equivalent circuit more physically meaningful; moreover, it improves the model accuracy for describing the low-frequency dispersion, as shown in Figure 12. This figure shows simulated frequency dispersion of the channel transconductance and output conductance, which is related mainly to the surface and buffer traps. The values of $\mathrm{R}_{\mathrm{GT}}, \mathrm{R}_{\mathrm{DT}}, \mathrm{C}_{\mathrm{GT}}$, and $\mathrm{C}_{\mathrm{DT}}$ are chosen to result in trapping time constants on the order of $10^{-5}-10^{-4} \mathrm{~s}$ (Meneghesso et al., 2001). In the current model, the amount of selfheating-induced current dispersion is controlled by normalized channel temperature rise $\Delta \mathrm{T}$. The normalized temperature rise is the channel temperature divided by the device thermal resistance $R_{\text {th }}$. A low-pass circuit is added to determine the value of $\Delta T$ due to the static and quasi-static dissipated power. The value of the thermal capacitance $C_{\text {th }}$ is selected to define a transit time constant on the order of $1 \mathrm{~ms}$ (Kohn et al., 2003). $R_{\text {th }}$ is normalized to one because its value is incorporated in thermal fitting parameter in the current-model expression, as will be discussed in section 4.2. 


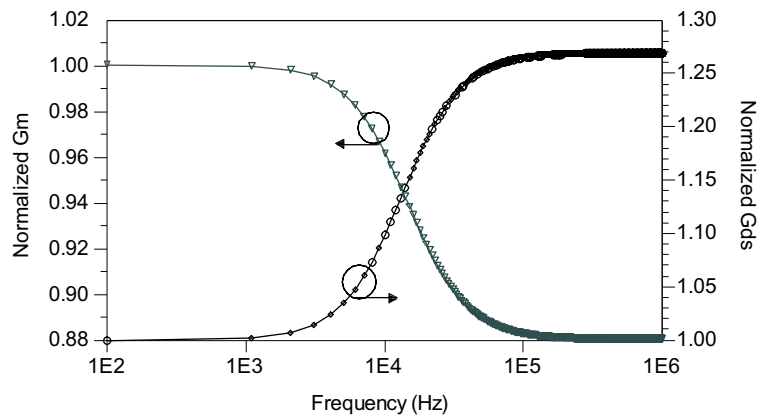

Fig. 12. Simulated normalized transconductance and output conductance for a $8 \times 125-\mu \mathrm{m}$ GaN HEMT at $V_{D S}=24 \mathrm{~V}$ and $V_{G S}=-2 \mathrm{~V}$.

\subsection{Gate charge and current modeling}

The intrinsic elements are extracted as a function of the extrinsic voltages $V_{G S}$ and $V_{D S}$ as presented in Figs. 6-8 for 2x50- $\mu \mathrm{m}$ GaN HEMT. To determine the intrinsic charge and current sources of the large-signal model by integration, a correction has to be carried out that considers the voltage drop across the extrinsic resistances. Therefore, the intrinsic voltages can be calculated as

$$
\begin{aligned}
& V_{d s}=V_{D S}-\left(R_{d}+R_{s}\right) I_{d s}-R_{s} I_{g s} \\
& V_{g s}=V_{G S}-\left(R_{g}+R_{s}\right) I_{g s}-R_{s} I_{d s} .
\end{aligned}
$$

This implies that the values of the intrinsic voltages $V_{g s}$ and $V_{d s}$ are no longer equidistant, which makes the intrinsic-element integration difficult to achieve. In addition, this representation is not convenient to handle in Advanced Design System (ADS) simulator. Interpolation technique can be used to uniformly redistribute the intrinsic element data with respect to equidistant intrinsic voltages. However, the main limitations of this technique are that it produces discontinuities and an almost oscillating behavior in the interpolated data. These effects result in inaccurate simulation of higher order derivatives of the current and charge sources, which deteriorate output-power harmonics and IMD simulations (Cuoco et al., 2002). Therefore, B-spline-approximation technique is used for providing a uniform data for the intrinsic elements (Jarndal \& Kompa, 2007). This technique can maintain the continuity of the data and its higher derivatives and hence improves the model simulation for the harmonics and the IMD (Koh et al., 2002). Generally, the intrinsic gate capacitances and conductances satisfy the integration path-independence rule (Root et al., 1991). Thus, the gate charges can be determined by integrating the intrinsic capacitances $\mathrm{C}_{\mathrm{gs}}, \mathrm{C}_{\mathrm{gd}}$, and $\mathrm{C}_{\mathrm{ds}}$ as follows (Schmale \& Kompa, 1997):

$$
Q_{g s}\left(V_{g s}, V_{d s}\right)=\int_{V_{g s 0}}^{V_{g s}} C_{g s}\left(V, V_{d s 0}\right) d V+\int_{V_{d s} 0}^{V_{d s}} C_{d s}\left(V_{g s}, V\right) d V
$$




$$
\begin{aligned}
Q_{g d}\left(V_{g s}, V_{d s}\right)= & \int_{V_{g s 0}}^{V_{g s}} C_{g d}\left(V, V_{d s o}\right) d V \\
& -\int_{V_{d s 0}}^{V_{d s}}\left[C_{d s}\left(V_{g s}, V\right)+C_{g d}\left(V_{g s}, V\right)\right] d V
\end{aligned}
$$

where $\mathrm{V}_{\mathrm{gs} 0}$ and $\mathrm{V}_{\mathrm{ds} 0}$ are arbitrary starting points for the integration. The shapes of the calculated $Q_{g s}$ and $Q_{g d}$, shown in Figure 13, for GaN HEMTs are similar to the reported ones for AlGaAs/GaAs HEMTs in (Schmale \& Kompa, 1997). The gate currents $I_{g s}$ and $I_{g d}$ are determined by the integration of the intrinsic gate conductances $G_{g f s}$ and $G_{g d f}$ as follows:

$$
\begin{gathered}
I_{g s}\left(V_{g s}, V_{d s}\right)=I_{g s}\left(V_{g s 0}, V_{d s 0}\right)+\int_{V_{g s 0}}^{V_{g s}} G_{g s f}\left(V, V_{d s 0}\right) d V \\
I_{g d}\left(V_{g s}, V_{d s}\right)= \\
I_{g d}\left(V_{g s 0}, V_{d s 0}\right)+\int_{V_{g s 0}}^{V_{g s}} G_{g d f}\left(V, V_{d s 0}\right) d V \\
\\
-\int_{V_{d s 0}}^{V_{d s}} G_{g d f}\left(V_{g s}, V\right) d V
\end{gathered} .
$$

The calculated values of $I_{g s}$ and $I_{g d}$ as a function of the intrinsic voltages are illustrated in Figure 14 .
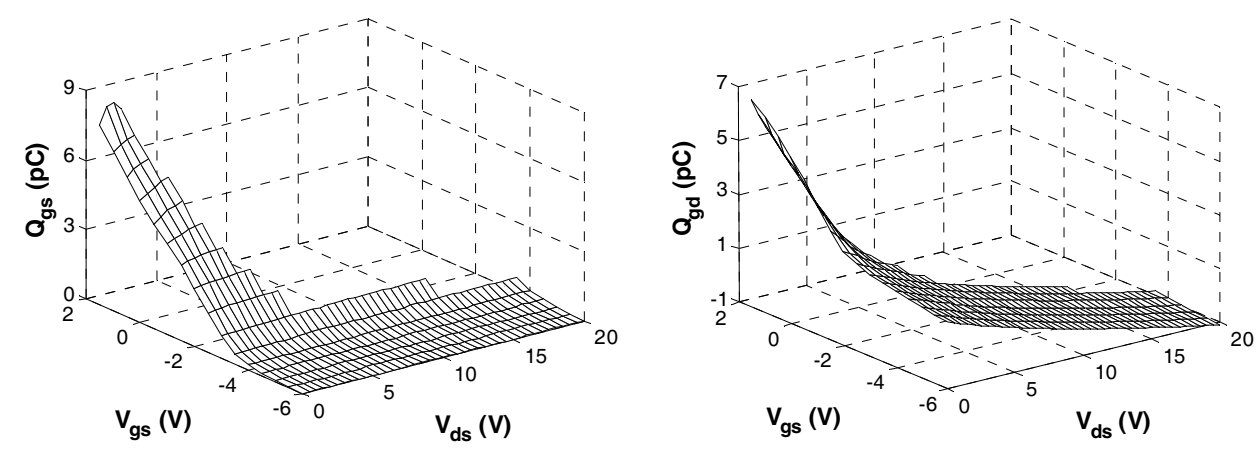

Fig. 13. Calculated gate-charge sources $Q_{g s}$ and $Q_{g d}$ versus intrinsic voltages for a $8 \times 125-\mu \mathrm{m}$ GaN HEMT. (C) 2007 IEEE. Reprinted with permission. 

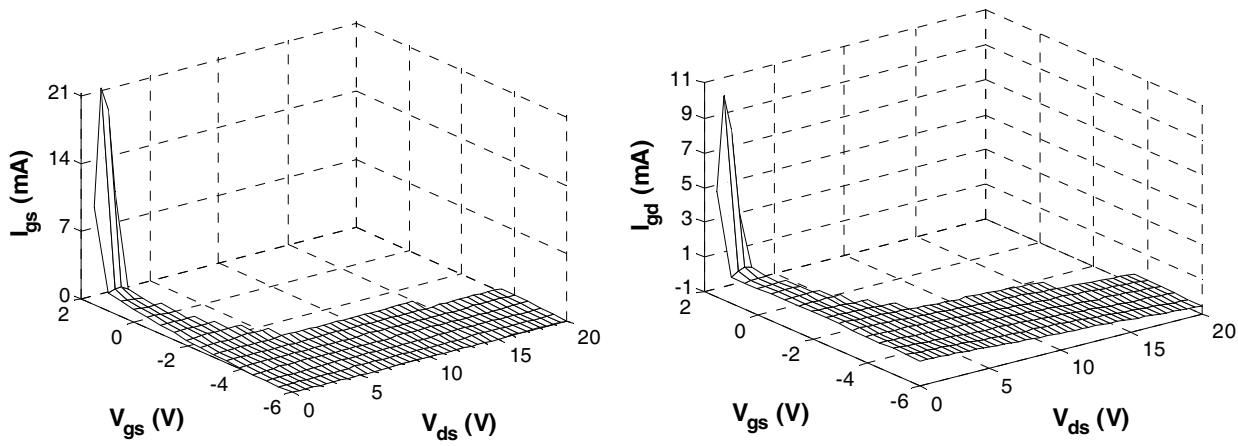

Fig. 14. Calculated gate-current sources $I_{g s}$ and $I_{g d}$ versus intrinsic voltages for a $8 \times 125-\mu m$ GaN HEMT. (c) 2007 IEEE. Reprinted with permission.

\subsection{Drain-current modeling}

Due to self-heating and trapping effects, associated with high-power devices, the intrinsic channel conductance and transconductance $\left(G_{d s}\right.$ and $\left.G_{m}\right)$ do not satisfy the integration pathindependence rule (Wei et al., 1999). Therefore, the RF drain current cannot be derived by relying on conventional S-parameter measurements. In addition, the self-heating and trapping cannot be characterized separately by these measurements to get an accurate current model. The optimal method is to derive the current model from pulsed I-V measurements under appropriate quiescent bias conditions, as presented in (Jarndalb et al., 2006). The drain current is modeled as (Filicori et al., 1995)

$$
\begin{aligned}
I_{d s}\left(V_{d s}, V_{g s}, V_{d s o}, V_{g s o}, P_{d i s s}\right)= & I_{d s, i s o}^{D C}\left(V_{g s}, V_{d s}\right) \\
& +\alpha_{G}\left(V_{g s}, V_{d s}\right)\left(V_{g s}-V_{g s o}\right) \\
& +\alpha_{D}\left(V_{g s}, V_{d s}\right)\left(V_{d s}-V_{d s o}\right) \\
& +\alpha_{T}\left(V_{g s}, V_{d s}\right) P_{d i s s}
\end{aligned}
$$

where $I D C_{d s, i s o}$ is the isothermal dc current after deembedding the self-heating effect. $\alpha_{G}$ and $\alpha_{D}$ model the deviation in the drain current due to the surface-trapping and buffer-trapping effects, respectively, and $\alpha_{T}$ models the deviation in the drain current due to the self-heating effect. The amount of trapping-induced current dispersion depends on the rate of dynamic change of the applied intrinsic voltages $V_{g s}$ and $V_{d s}$ with respect to those average values $V_{g s o}$ and $V_{d s o}$. In other words, this current dispersion is mainly stimulated by the RF or the ac components of the gate-source and drain-source voltages, which is described by $\left(V_{g^{s}}-V_{g_{s o}}\right)$ and $\left(V_{d s}-V_{d s o}\right)$ in (48). The self-heating-induced dispersion is caused mainly by the lowfrequency components of the drain signal. Therefore, $P_{\text {diss }}$ in (48) accounts for the static and quasistatic intrinsic power dissipation.

\section{A. Trapping and self-heating characterization}

Trapping effects can be characterized by pulsed I-V measurements at negligible device selfheating (Charbonniaud et al., 2003). The surface trapping is characterized by pulsed I-V's at two extrinsic quiescent biases equivalent to: 


$$
\begin{gathered}
V_{G S O}<V_{P}, V_{D S O}=0 V\left(P_{\text {diss }} \approx 0\right) \\
V_{G S O}=0 V, V_{D S O}=0 V\left(P_{\text {diss }} \approx 0\right) .
\end{gathered}
$$

The buffer trapping is characterized by pulsed I-V's at two quiescent biases equivalent to:

$$
\begin{gathered}
V_{G S O}<V_{P}, V_{D S O}=0 V\left(P_{\text {diss }} \approx 0\right) \\
V_{G S O}<V_{P}, V_{D S O}>0 \quad V\left(P_{\text {diss }} \approx 0\right) .
\end{gathered}
$$

These two conditions lead to different states of the trapping effects but involve negligible power dissipation. To characterize the self-heating, additional pulsed I-V characteristics at rather high quiescent power dissipation are used. DC I-V characteristics can also be used in addition to the pulsed I-V characteristics for further improvement of the self-heating characterization (Jarndalb et al., 2006).

\section{B. Drain-current-model parameter extraction}
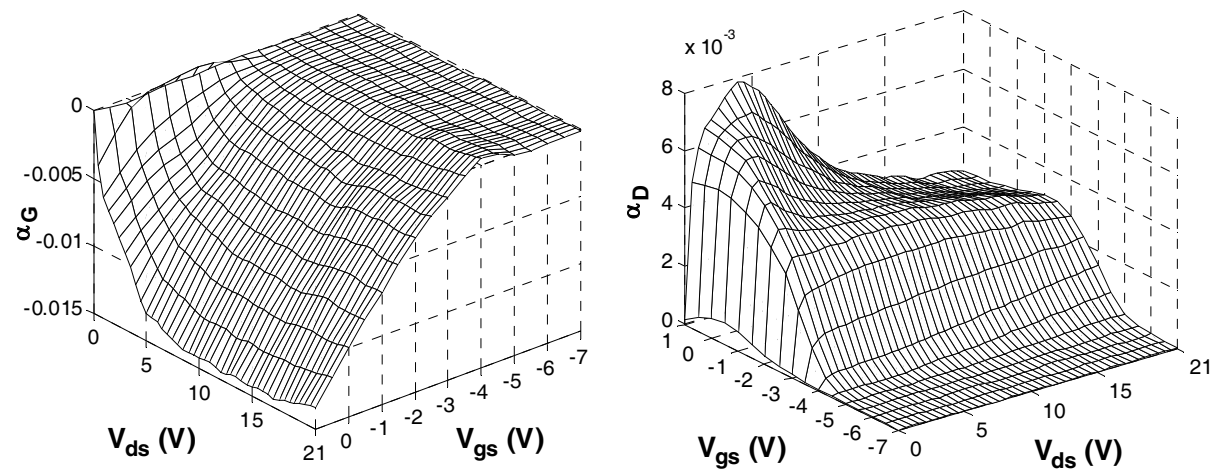

Fig. 15. Bias-dependent trapping fitting parameters of the drain-current model in (48) extracted from the pulsed I-V measurements of a $8 \times 125-\mu \mathrm{m}$ GaN HEMT. (c) 2007 IEEE. Reprinted with permission.

The drain-current-model equation in (48) has four unknowns: $I D C_{d s, i s o,} a_{G}, a_{D}$, and $a_{T}$. To determine these unknowns, the equation should be applied to, at least, four pulsed I-V characteristics at suitable quiescent bias conditions that lead to four highly independent linear equations. The described I-V characteristics in Section 4.2-A define approximately four independent states for the drain current. At each state, the drain current can be assumed to be affected by, at most, one of the dispersion sources (surface trapping, buffer trapping, or self-heating). By solving the four linear equations, corresponding to the four characteristics, at each bias point, the values of $I D C_{d s, i s o}, a_{G}, a_{D}$, and $a_{T}$ can be determined. Figs. 15 and 16 show the extracted values of these fitting parameters as a function of the intrinsic voltages. 


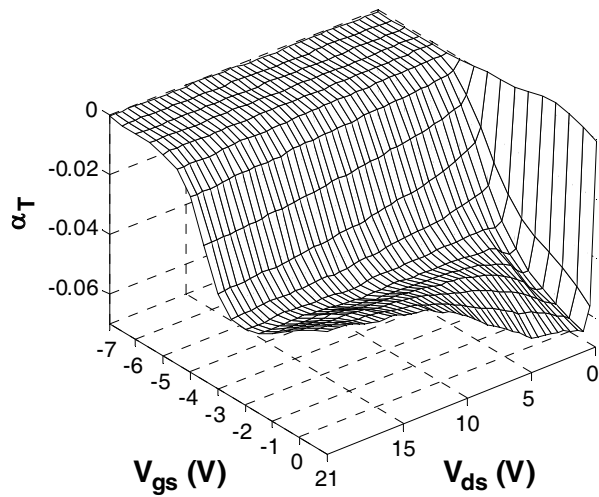

(a)

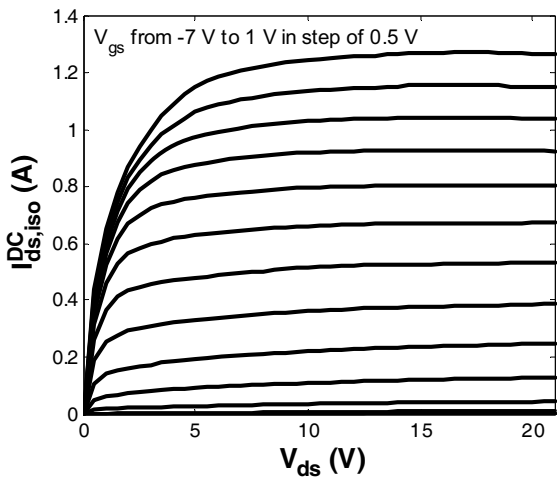

(b)

Fig. 16. (a) Extracted bias-dependent self-heating fitting parameter and (b) isothermal dc drain current for a $8 \times 125-\mu \mathrm{m}$ GaN HEMT. (C) 2007 IEEE. Reprinted with permission.

\subsection{Large-signal model implementation and verification}

The large-signal model was implemented as a table-based model in ADS. The extrinsic biasindependent passive elements are represented by lumped elements, whereas the intrinsic nonlinear part is represented by a symbolically defined device (SDD) component.

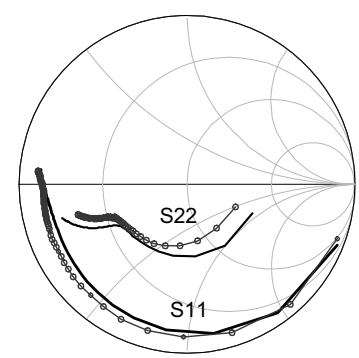

freq $(150.0 \mathrm{MHz}$ to $20.00 \mathrm{GHz})$

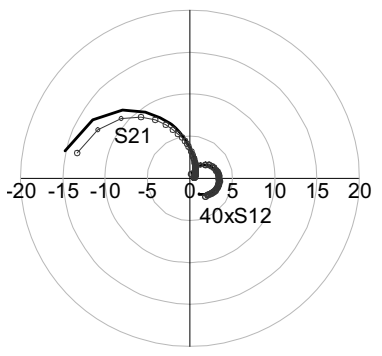

freq $(150.0 \mathrm{MHz}$ to $20.00 \mathrm{GHz})$

(a)

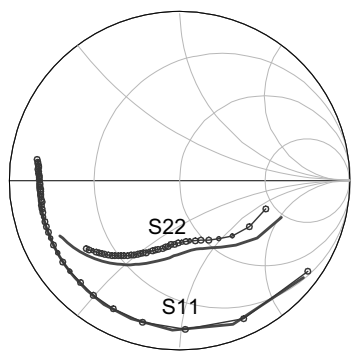

freq $(500.0 \mathrm{MHz}$ to $20.00 \mathrm{GHz})$

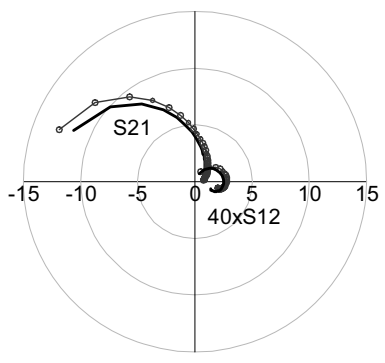

freq $(500.0 \mathrm{MHz}$ to $20.00 \mathrm{GHz})$

(b)

Fig. 17. (Lines) Simulated and (circles) measured S-parameters of a 8x125- $\mu \mathrm{m}$ GaN HEMT at (a) $\mathrm{V}_{\mathrm{GS}}=-2.0 \mathrm{~V}$ and $\mathrm{V}_{\mathrm{DS}}=9.0 \mathrm{~V}$ and (b) $\mathrm{V}_{\mathrm{GS}}=-3.0 \mathrm{~V}$ and $\mathrm{V}_{\mathrm{DS}}=21.0 \mathrm{~V}$. 
The developed large-signal model was verified by independent measurements. The considered devices are $8 \times 125-\mu \mathrm{m}$ GaN HEMTs on different wafers. First, the model is checked whether it is consistent with I-V and S-parameter measurements it has been derived from. Second, large-signal single- and two-tone simulations are compared with measurements. S-parameter simulation in comparison with measurement of a $8 \times 125-\mu \mathrm{m}$ device is shown in Figure 17. The good agreement between simulation and measurement verifies the consistency of the large-signal model with the small-signal equivalent-circuit model. Pulsed I-V simulation has been done at quiescent bias conditions different than the used ones for model parameter extraction. Figure 18 shows pulsed I-V simulations on two different quiescent bias conditions at constant ambient temperature.
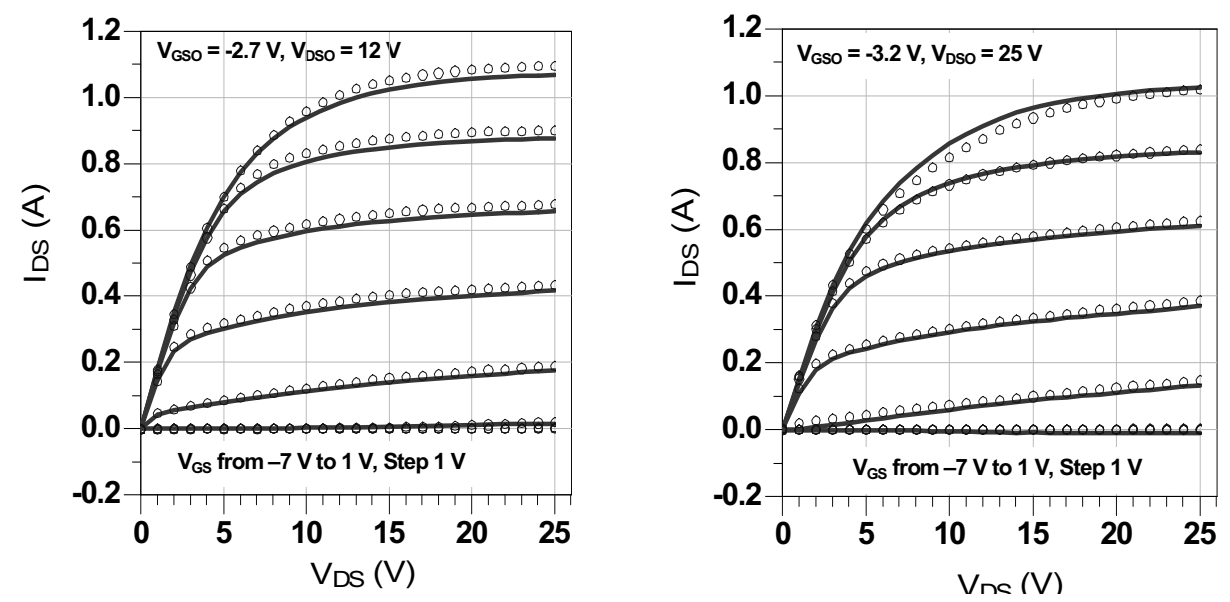

Fig. 18 (Lines) Pulsed I-V simulations and (circles) measurements for a $8 \times 125-\mu \mathrm{m}$ GaN HEMT at different quiescent bias conditions. (C) 2006 IEEE. Reprinted with permission.
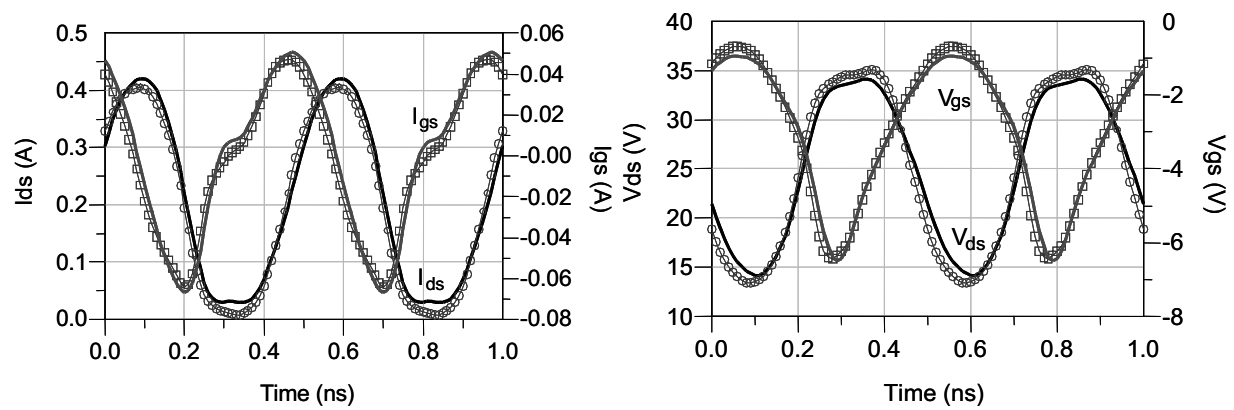

Fig. 19 (Lines) Simulated and (symbols) measured large-signal waveforms for class-ABoperated 8x125- $\mu \mathrm{m}$ GaN HEMT at 16-dBm input power. (C) 2006 IEEE. Reprinted with permission.

The very good agreement between simulation and measurement shows the ability of the model for describing the bias dependence of the trapping and self-heating effects. In 
addition, these simulations verify the convergence behaviour of the model response under pulsed stimulation, which is very important for digital applications. Large-signal waveform measurements for $8 \times 125-\mu \mathrm{m}$ GaN HEMTs were done using the measurement setup described in (Raay \& Kompa, 1997) and then simulated by the model. As it can be seen in Figure 19, very good agreement between measured and simulated current and voltage waveforms is obtained. This can be related to the improved construction of the model elements using the spline-approximation technique, as explained in Section 4.1, which improves the modeling of the higher order harmonics.
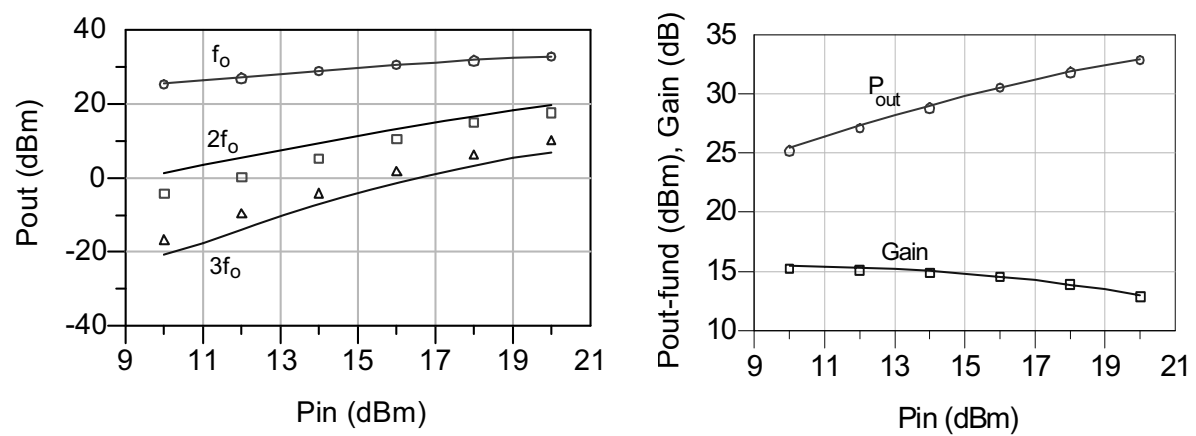

Fig. 20. (Lines) Single-tone power-sweep simulations compared with (symbols) measurements for class-A-operated $8 \times 125-\mu \mathrm{m}$ GaN HEMT at $2 \mathrm{GHz}$ in a $50-\Omega$ source and load environment. ( 2007 IEEE. Reprinted with permission.

Figure 20 shows a simulation result of a single-tone input-power sweep for a $8 \times 125-\mu \mathrm{m} \mathrm{GaN}$ HEMT. The model shows very good results with respect to the fundamental output power and gain even for input-power levels beyond the 1-dB gain-compression point. The model also shows good simulation results for the output power of higher harmonic components up to the third harmonic.
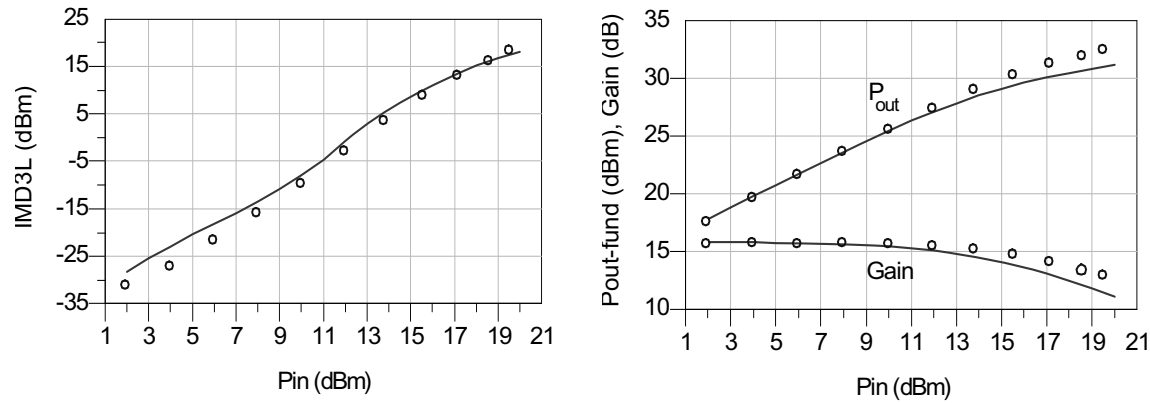

Fig. 21. (Lines) Simulated and (symbols) measured Pout, Gain and IMD3 versus input power per tone under two-tone excitation centered at $2 \mathrm{GHz}$ and separated by $100 \mathrm{kHz}$ for class-AB-operated 8x125- $\mu \mathrm{m}$ GaN HEMT in a 50- $\Omega$ source and load environment. (C) 2007 IEEE. Reprinted with permission. 
Simulations for output power, gain and third intermodulation distortion under two-tone excitation centered at $2 \mathrm{GHz}$ and separated by $100 \mathrm{kHz}$ were performed. The simulation results are compared with measurements of $8 \times 125-\mu \mathrm{m}$ GaN HEMTs on different wafers. These measurements were performed using the developed measurement setups described in (Ahmad et al., 2005). Figure 21 presents the simulation results in comparison with the measurements. The model shows very good results for describing the output power and gain except at high-power end. The inaccuracy is due to the extrapolation error outside the region of measurements where the model was derived from. The model accuracy can be improved by increasing the range of these measurements to cover higher voltage conditions. The model also shows very good simulation for the third-order IMD. This can also be related to the use of spline approximation for the construction of the model-element data.
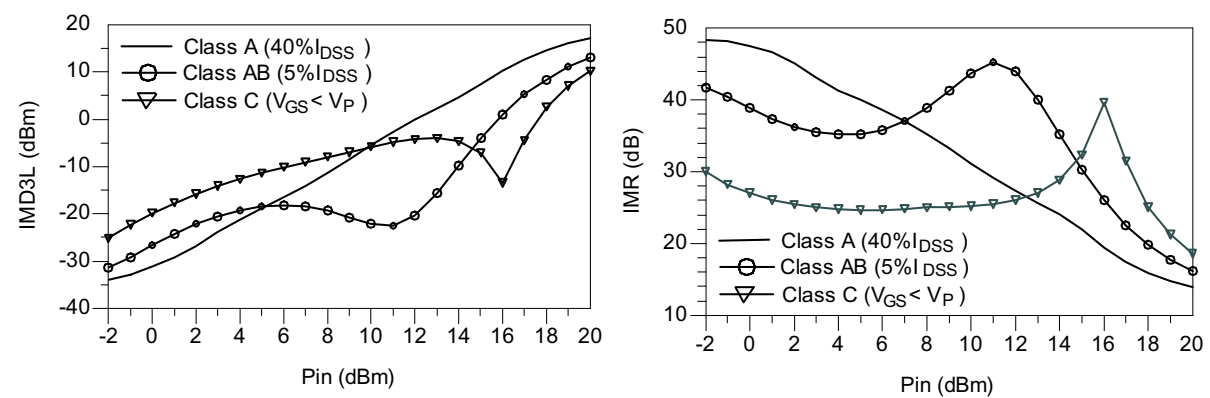

Fig. 22 Simulated lower intermodulation distortion and carrier to intermodulation ratio versus input power per tone under two-tone excitation centered at $2 \mathrm{GHz}$ and separated by $100 \mathrm{kHz}$ for a $8 \times 125-\mu \mathrm{m} \mathrm{GaN} \mathrm{HEMT} \mathrm{under} 20 \mathrm{~V}$ drain bias voltage for different gate bias voltages in a $50 \Omega$ source and load environment.

Figure 22 shows simulated lower IMD3 and the corresponding carrier to intermodulation ratio (IMR) for $8 \times 125 \mu \mathrm{m}$ GaN HEMT under two-tone excitation for different classes of operation. The model shows very good results for prediction of the IMD3 sweet spots (local minima), which result from the interaction between small- and large signal IMDs (Carvalho \& Pedro, 1999; Fager et al., 2002). The IMD3 simulation is done at different gate bias conditions for $20 \mathrm{~V}$ drain biased device in a $50-\Omega$ source and load environment. It is found that the best performance with maximum IMR and high power added efficiency could be obtained when the device is biased just above the pinch-off voltage as illustrated in Figure 22. These results are in a very good agreement with the reported ones in (Cabral et al., 2004) for a 2-mm gate width GaN HEMT.

\section{Conclusion}

In this chapter, a large-signal model for GaN HEMTs, which accurately predicts trappingand self-heating-induced current dispersion and IMD, was developed and demonstrated. Detailed procedures for both small-signal and large-signal model parameter extraction has been presented. The extracted intrinsic gate capacitances and conductances of distributed small-signal model were integrated to find the gate charge and current sources of the largesignal model, assuming that these elements satisfy the integral path-independence 
condition. Pulsed I-V measurements under appropriate quiescent bias conditions were used to accurately characterize and model the drain current and the inherent self-heating and trapping effects. It is found that using approximation technique for the construction of the large-signal-model database can improve the model capability for harmonics and IMD simulations. Large-signal simulations show that the model can accurately describe the performance of the device under constant external temperature. However, this model can also be extended to consider the variation of the ambient temperature.

\section{References}

Ambacher, O.; Smart, J.; Shealy, J.; Weimann, N.; Chu, K.; Murphy, M.; Schaff, W.; Eastman, L.; Dimitrov, R.; Wittmer, L.; Stutzman, M.; Rieger, W. and Hilsenbeck, J. (1999). Two-dimensional electron gases induced by spontaneous and piezoelectric polarization in undoped and doped AlGaN/GaN heterostructures. Journal of Applied Physics, Vol. 85, (March 1999) page numbers (3222-3232), ISSN 0021-8979.

Ahmed, A.; Srinidhi, E. \& Kompa, G. (2005). Efficient PA modeling using neural network and measurement set-up for memory effect characterization in the power device, WE1D-5, ISBN 0-7803-8845-3, Proceeding of International Microwave Symposium Digest, USA, June 2005, Long Beach.

Cabral, P.; Pedro, J. \& Carvalho, N. (2004). Nonlinear device model of microwave power GaN HEMTs for high power amplifier design. IEEE Transaction Microwave Theory and Techniques, Vol. 52, (November 2004) page numbers (2585-2592), ISSN 00189480.

Cuoco, V.; Van den Heijden, M. \& De Vreede, L. (2002). The 'smoothie' data base model for the correct modeling of non-linear distortion in FET devices, Proceeding of International Microwave Symposium Digest, pp. 2149-2152, ISBN 0-7803-7239-5, USA, February 2002, IEEE, Seattle.

Charbonniaud, C.; De Meyer, S.; Quere, R. \& Teyssier, J. (2003). Electrothermal and trapping effects characterization of AlGaN/GaN HEMTs, Proceeding of European Gallium Arsenide E related III-V Compounds Application Symposium, pp. 201-204, ISBN 158053-837-1, Germany, October 2003, Munich.

Carvalho, N. \& Pedro, J. (1999). Large-and small-signal IMD behavior of microwave power amplifier. IEEE Transaction Microwave Theory and Techniques, Vol. 47, (December 1999) page numbers (2364-2374), ISSN 0018-9480.

Edwards, M. and Sinsky, J. (1992). A new criterion for linear two-port stability using a single geometrically derived parameter. IEEE Transaction Microwave Theory and Techniques, Vol. 40, (December 1992) page number (2303-2311), ISSN 0018-9480.

Eastman, L.; Tilak, V.; Smart, J.; Green, B.; Chumbes, E.; Dimitrov, R.; Hyungtak, K.; Ambacher, O; Weimann, N; Prunty, T; Murphy, M.; Schaff, W. \& Shealy, J. (2001). Undoped GaN HEMTs for microwave power amplification. IEEE Transaction on Electron Devices, Vol. 48, (March 2001) page numbers (479-485), ISSN 0018-9383.

Filicori, F.; Vannini, G.; Santarelli, A.; Mediavilla, A.; Tazón, A. \& Newport, Y. (1995). Empirical modeling of low-frequency dispersive effects due to traps and thermal phenomena in III-V FETs. IEEE Transaction Microwave Theory and Techniques, Vol. 43, (December 1995) page numbers (2972-2981), ISSN 0018-9480. 
Fager, C.; Pedro, J.; Carvalho, N. \& Zirath, H. (2002). Prediction of IMD in LDMOS transistor amplifiers using a new large-signal model, IEEE Transaction Microwave Theory and Techniques, Vol. 50, (December 2002) page numbers (2834-2842), ISSN 0018-9480.

Green, B.; Chu, K.; Kim, H.; Lin, H.; Tilak, V.; Shealy, J.; Smart, A. \& Eastman, L. (2000). Validation of an analytical large signal model for GaN HEMTs, Proceeding of International Microwave Symposium Digest, pp. 761-764, ISBN 0-7803-5687-X, USA, June 2000, IEEE, Boston.

Goyal, R. (1989). Monolithic Microwave Integrated Circuit: Technology and Design, Artech House, ISBN 0890063095, Norwood, USA.

Hansen, P.; Strausser, Y.; Erickson, A.; Tarsa, E.; Kozodoy, P.; Brazel, E.; Ibbetson, J.; Mishra, U.; Narayanamurti, V.; DenBaars, S.; and Speck, J. (1998). Scanning capacitance microscopy imaging of threading dislocations in GaN films grown on (0001) sapphire by metalorganic chemical vapor deposition. Applied Physics Letters, Vol. 72, (May 1998) page numbers (2247-2249), ISSN 0003-6951.

Jarndal, A. \& Kompa, G. (2005). A new small-signal modeling approach applied to GaN devices. IEEE Transaction Microwave Theory and Techniques, Vol. 53, (November 2005), page numbers (3440-3448), ISSN 0018-9480.

Jarndala, A. \& Kompa, G. (2006). An accurate small-signal model for AlGaN-GaN HEMT suitable for scalable large-signal model construction. IEEE Microwave and Wireless Components Letters, Vol. 16, (June 2006) page numbers (333-335), ISSN 1531-1309.

Jarndalb, A.; Bunz, B. \& Kompa, G. (2006). Accurate large-signal modeling of AlGaN-GaN HEMT including trapping and self-heating induced dispersion, Proceeding IEEE International Symposium Power Semiconductor Devices and ICs, pp. 1-4, ISBN 0-78039714-2, Italy, June 2006, Napoli.

Jarndal, A. \& Kompa, G. (2007). Large-Signal Model for AlGaN/GaN HEMT Accurately Predicts Trapping and Self-Heating Induced Dispersion and Intermodulation Distortion. IEEE Transaction Microwave Theory and Techniques, Vol. 54, (November 2007) page numbers (2830-2836), ISSN 0018-9480.

Kohn, E.; Daumiller, I.; Kunze, M.; Neuburger, M.; Seyboth, M.; Jenkins, T.; Sewell, J.; Norstand, J.; Smorchkova, Y. \& Mishra, U. (2003). Transient characteristics of GaNbased heterostructure field-effect transistors. IEEE Transaction Microwave Theory and Techniques, Vol. 51, (Februry 2003) page numbers (634-642), ISSN 0018-9480.

Koh, K.; Park, H.-M. \& Hong, S. (2002). A spline large-signal FET model based on biasdependent pulsed I-V measurements. IEEE Transaction Microwave Theory and Techniques, Vol. 50 (November 2002) page numbers (2598-2603), ISSN 0018-9480.

Kotzebue, K. (1976). Maximally Efficient Gain: A Figure of Merit for Linear Active 2-Ports. Electronics Letters, Vol. 12, (September 1976) page numbers (490-491), ISSN 00135194.

Kompa, G. \& Novotny, M. (1997). Frequency-dependent measurement error analysis and refined FET model parameter extraction including bias-dependent series resistors, Proceeding of International IEEE Workshop on Experimentally Based FET Device Modelling and Related Nonlinear Circuit Design, pp. 6.1-6.16, Report Number: A864133, University of Kassel, Germany, July 1997, IEEE, Kassel.

Lossya, R.; Chaturvedi, N.; Heymann, P.; Würfl, J.; Müller, S.; and Köhler, K. (2002). Large area AlGaN/GaN HEMTs grown on insulating silicon carbide substrates. Physica Status Solidi (a), Vol. 194, (December 2002) page numbers (460-463), ISSN 0031-8965. 
Lossy, R.; Hilsenbeck, J.; Würfl, J. and Obloh, H. (2001). Uniformity and scalability of AlGaN/GaN HEMTs using stepper lithography. Physica Status Solidi (a), Vol. 188, (November 2001) page numbers (263-266), ), ISSN 0031-8965.

Lossyb, R.; Heymann, P.; Würfl, J.; Chaturvedi, N.; Müller, S.; and Köhler, K. (2002). Power RF-operation of AlGaN/GaN HEMTs grown on insulating silicon carbide substrates, Proceeding of European Gallium Arsenide $\mathcal{E}$ related III-V Compounds Application Symposium, ISBN 0-86213-213-4, Italy, September 2002, IEEE, Milan.

Lee, J. \& Webb, K. (2004). A temperature-dependent nonlinear analytic model for AlGaNGaN HEMTs on SiC. IEEE Transaction Microwave Theory and Techniques, Vol. 52, (January 2004) page numbers (2-9), ISSN 0018-9480.

Lin, L. \& Kompa, G. (1994). FET model parameter extraction based on optimization with multiplane data-fitting and bidirectional search-A new concept. IEEE Transaction Microwave Theory and Techniques, Vol. 42, (July 1994) page numbers (1114-1121), ISSN 0018-9480.

Lu, S.-S.; Chen, T.-W.; Chen, H.-C.; Meng, C. (2001). The origin of the kink phenomenon of transistor scattering parameter $\mathrm{S}_{22}$. IEEE Transaction Microwave Theory and Techniques, Vol. 49, (February 2001) page numbers (333 - 340), ISSN 0018-9480.

Meneghesso, G.; Verzellesi, G. ; Pierobon, R. ; Rampazzo, F.; Chini, A.; Mishra, U.; Canali, C. \& Zanoni, E. (2004). Surface-related drain current dispersion effects in AlGaN-GaN HEMTs. IEEE Transaction Microwave Theory and Techniques, Vol. 51, (October 2004) page numbers (1554-1561), ISSN 0018-9480.

Root, D.; Fan, S. \& Meyer, J. (1991). Technology Independent Large Signal Non Quasi-Static FET Models by Direct Construction from Automatically Characterized Device Data, Proceeding of European Microwave Conference, pp. 927 - 932, EUMA.1991.336465, Germany, October 1991, IEEE, Stuttgart.

Raay, F.; Quay, R.; Kiefer, R.; Schlechtweg, M. \& Weimann, G. (2003). Large signal modeling of GaN HEMTs with Psat $>4 \mathrm{~W} / \mathrm{mm}$ at $30 \mathrm{GHz}$ suitable for broadband power applications, Proceeding of International Microwave Symposium Digest, USA, PA, pp. 451-454, ISBN 0-7803-7695-1, June 2003, IEEE, Philadelphia.

Schmale, I. \& Kompa, G. (1997). A physics-based non-linear FET model including dispersion and high gate-forward currents, Proceeding of International Workshop on Experimentally Based FET Device Modelling and Related Nonlinear Circuit Design, pp. 27.1-27.7, Report Number: A864133, University of Kassel, Germany, July 1997, IEEE, Kassel.

Schmale, I. \& Kompa, G. (1997). An improved physics-based nonquasistatic FET-model, Proceeding of European Microwave Conference, pp. 328-330, ISBN 0-7803-4202-X, Jerusalem, September 1997, IEEE, Israel.

System Manual HP8510B Network Analyzer (1987). HP Company, P/N 08510-90074, USA, Santa Rosa, July 1987.

Vetury, R; Zhang, N; Keller, S. \& Mishra, U. (2001). The impact of surface states on the DC and RF characteristics of GaN HFETs. IEEE Transaction on Electron Devices, Vol. 48, (March 2001) page numbers (560-566), ISSN 0018-9383.

Van Raay, F. \& Kompa, G. (1997). Combination of waveform and load-pull measurements, Proceeding of International IEEE Workshop on Experimentally Based FET Device Modelling and Related Nonlinear Circuit Design, pp. 10.1-10.11, Report Number: A864133, University of Kassel, Germany, July 1997, IEEE, Kassel. 
Wei, C.; Tkachenko, Y. \& Bartle, D. (1999). Table-based dynamic FET model assembled from small-signal models. IEEE Transaction Microwave Theory and Techniques, Vol. 47, (June 1999) page numbers (700-705), ISSN 0018-9480. 


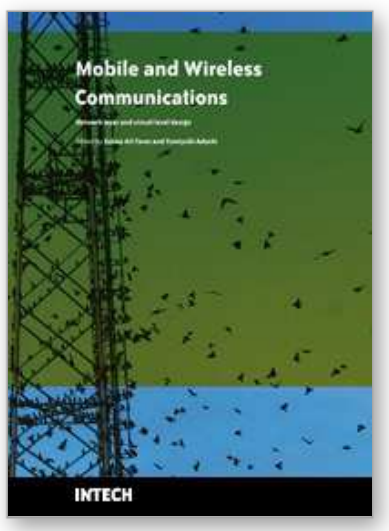

\section{Mobile and Wireless Communications Network Layer and Circuit Level Design}

Edited by Salma Ait Fares and Fumiyuki Adachi

ISBN 978-953-307-042-1

Hard cover, 404 pages

Publisher InTech

Published online 01, January, 2010

Published in print edition January, 2010

Mobile and wireless communications applications have a clear impact on improving the humanity wellbeing. From cell phones to wireless internet to home and office devices, most of the applications are converted from wired into wireless communication. Smart and advanced wireless communication environments represent the future technology and evolutionary development step in homes, hospitals, industrial, vehicular and transportation systems. A very appealing research area in these environments has been the wireless ad hoc, sensor and mesh networks. These networks rely on ultra low powered processing nodes that sense surrounding environment temperature, pressure, humidity, motion or chemical hazards, etc. Moreover, the radio frequency (RF) transceiver nodes of such networks require the design of transmitter and receiver equipped with high performance building blocks including antennas, power and low noise amplifiers, mixers and voltage controlled oscillators. Nowadays, the researchers are facing several challenges to design such building blocks while complying with ultra low power consumption, small area and high performance constraints. CMOS technology represents an excellent candidate to facilitate the integration of the whole transceiver on a single chip. However, several challenges have to be tackled while designing and using nanoscale $\mathrm{CMOS}$ technologies and require innovative idea from researchers and circuits designers. While major researchers and applications have been focusing on RF wireless communication, optical wireless communication based system has started to draw some attention from researchers for a terrestrial system as well as for aerial and satellite terminals. This renewed interested in optical wireless communications is driven by several advantages such as no licensing requirements policy, no RF radiation hazards, and no need to dig up roads besides its large bandwidth and low power consumption. This second part of the book, Mobile and Wireless Communications: Key Technologies and Future Applications, covers the recent development in ad hoc and sensor networks, the implementation of state of the art of wireless transceivers building blocks and recent development on optical wireless communication systems. We hope that this book will be useful for students, researchers and practitioners in their research studies.

\section{How to reference}

In order to correctly reference this scholarly work, feel free to copy and paste the following:

Anwar Jarndal (2010). Large-Signal Modeling of GaN Devices for Designing High Power Amplifiers of Next Generation Wireless Communication Systems, Mobile and Wireless Communications Network Layer and Circuit Level Design, Salma Ait Fares and Fumiyuki Adachi (Ed.), ISBN: 978-953-307-042-1, InTech, Available from: http://www.intechopen.com/books/mobile-and-wireless-communications-network-layer-and-circuit-leveldesign/large-signal-modeling-of-gan-devices-for-designing-high-power-amplifiers-of-next-generation-wireless 
open science | open minds

\section{InTech Europe}

University Campus STeP Ri

Slavka Krautzeka 83/A

51000 Rijeka, Croatia

Phone: +385 (51) 770447

Fax: +385 (51) 686166

www.intechopen.com

\section{InTech China}

Unit 405, Office Block, Hotel Equatorial Shanghai

No.65, Yan An Road (West), Shanghai, 200040, China

中国上海市延安西路65号上海国际贵都大饭店办公楼 405 单元

Phone: +86-21-62489820

Fax: +86-21-62489821 
(C) 2010 The Author(s). Licensee IntechOpen. This chapter is distributed under the terms of the Creative Commons Attribution-NonCommercial-ShareAlike-3.0 License, which permits use, distribution and reproduction for non-commercial purposes, provided the original is properly cited and derivative works building on this content are distributed under the same license. 